\title{
On a Fourier Expansion in Continuous Crossed Products
}

By

\author{
Hiroshi TAKAI*
}

\begin{abstract}
Let $(M, \mathbb{R}, \alpha)$ be a separable continuous $W^{*}$-dynamical system such that $M$ is $\mathbb{R}$-finite.

Then any element in the crossed product $\mathbb{R} \otimes_{\alpha} M$ of $M$ by $\alpha$ can be expressed as a vector valued tempered distribution $D^{q} T_{\eta}$ which, is a weak* limit of $T_{\xi}, \xi \in K(\mathbb{R} ; \mathfrak{B})$ in the dual space $S_{U}(\mathbb{R} ; \mathfrak{y})^{*}$ of a generalized Schwartz space $S_{U}(\mathbb{R} ; \mathfrak{y})$, where $K(\mathbb{R} ; \mathfrak{B})$ is the Tomita algebra corresponding to $\mathbb{R} \otimes_{\alpha} M$.
\end{abstract}

\section{$\S 1$. - nutroduction}

The study of von Neumann algebra of type III has been greatly developed since M. Tomita [10] obtained the so-called commutation theorem based on his deep ideas. A. Connes [1] classified factors of type III into three parts - that is, of type $\mathbb{I I}_{0}$, of type $\operatorname{III}_{\lambda}(0<\lambda<1)$ and of type $\mathrm{III}_{1}-$, and he proved that a factor of type $\mathrm{III}_{0}$ or $\mathrm{II}_{\lambda}$ $(0<\lambda<1)$ is the crossed product of a von Neumann algebra of type $\mathbb{I I}_{\infty}$ by a single automorphism. Independently, $M$. Takesaki [14] and $H$. Araki [13] found that certain class of type III factors are the crossed products of von Neumann algebras of type $\mathrm{II}_{1}$ by a single automorphisms. M. Takesaki [9], using a duality for crossed products, established a structure theorem that any factor of type III with separable predual can be written as the crossed product of a von Neumann algebra of type $\mathrm{II}_{\infty}$ by a continuous action of the real numbers. A. Connes [2] verified that there exist factors of type III which are isomorphic to no discrete crossed product of a semifinite von Neumann algebra by an

Communicated by H. Araki, September 26, 1975.

* Department of Mathematical Sciences, Osaka University, Toyonaka, Japan. 
abelian group.

These facts tell us that discrete crossed products can hardly cover all the structures of factors of type III. Therefore, it is quite significant to study continuous crossed products systematically.

However, there appear a lot of complications in the continuous case as compared with the discrete case. For instance, it is unclear whether the primitive ideal space of crossed products equals to the induced primitive ideal space in separable continuous $C^{*}$-dynamical systems. (cf: [8])

The main reason for the difference seems to come from a lack of a suitable way to express any element in continuous crossed products as an operator valued function with certain rules.

From this point of view, it is desirable to find a proper Fourier expansion in continuous crossed products as in the discrete case.

In this paper, we shall try to offer one version for Fourier expansions as stated above. More precisely, any element in the crossed product $\mathbb{R} \otimes_{\alpha} M$ of a $\mathbb{R}$-finite von Neumann algebra $M$ with the separable predual $M_{*}$ by a continuous action $\alpha$ of the real numbers $\mathbb{R}$, can be expressed as a vector valued tempered distribution $D^{q} T_{\eta}$ which is a weak* limit of $T_{\zeta}, \zeta \in K(\mathbb{R} ; \mathfrak{B})$ in the dual space $S_{U}(\mathbb{R} ; \mathfrak{y})^{*}$ of a generalized Schwartz space $S_{U}(\mathbb{R} ; \mathfrak{y})$, where $K(\mathbb{R} ; \mathfrak{B})$ is the Tomita algebra corresponding to $\mathbb{R} \otimes_{\alpha} M$.

Finally, the author would like to express his hearty thanks to Professor $\mathrm{O}$. Takenouchi for his constant encouragement and warm hospitality while this manuscript is being prepared.

$\mathrm{He}$ also is greatly indebted to Professor A. Connes for his careful reading of this paper and many valuable suggestions, and to Professor H. Araki for his kind advice.

\section{§2. Continuous $\mathbb{W}^{*}$-Crossed Products and Preliminary Lemmas}

In this section, we shall define the crossed product associated with a separable continuous $W^{*}$-dynamical system and prepare a couple of lemmas which will be used later. Let $M$ be a von Neumann algebra and $G$ be a locally compact group. Consider a mapping $\alpha$ of $G$ into the group $\operatorname{Aut}(M)$ of all automorphisms of $M$. The triple $(M, G, \alpha)$ 
is said to be a continuous $W^{*}$-dynamical system if $\alpha$ is a homomorphism such that the function $g \mapsto \phi \circ \alpha_{g}(x)$ is continuous on $G$ for every $x \in M$ and $\phi \in M_{*}$, where $M_{*}$ is the predual of $M$. It is also called separable if $M_{*}$ and $G$ are separable. Let us suppose from now on that a triple $(M, G, \alpha)$ is a separable continuous $W^{*}$-dynamical system and $G$ is unimodular. Comparing with the discrete case, one more assumption would be added as follows: There exists a $G$-invariant faithful normal state $\phi$ on $M$. Namely, suppose that $M$ is $G$-finite. Associated with $\phi$, let $\mathfrak{Q}_{\phi}, \mathfrak{B}, \Delta, J$ be the full left Hilbert algebra with the identity $\xi_{0}$, the maximal Tomita algebra in $\mathfrak{A}_{\phi}$, the modular operator of $\mathfrak{A}_{\phi}$, the unitary involution of $\mathfrak{H}_{\phi}$ respectively. Since $\phi$ is $G$-invariant, there exists a strongly continuous unitary representation $U$ of $G$ on a Hilbert space $\mathfrak{y}$ such that $U_{g} \eta_{\phi}(x)=\eta_{\phi} \circ \alpha_{g}(x)$ for all $x \in M$, where $\eta_{\phi}$ is the canonical imbedding of $M$ into $\mathfrak{y}$. Since $\Delta$ commutes with $U_{g}, \mathfrak{B}$ is invariant under $U_{g}$. According to M. Takesaki [9], a locally convex topology on $\mathfrak{B}$ is defined by the following system $\left(P_{K}\right)_{K}$ of seminorms:

$$
P_{K}(\xi)=\sup _{z \in K}\left\{\left\|\Pi_{l}\left(\Delta^{z} \xi\right)\right\|+\left\|\Pi_{r}\left(\Delta^{z} \xi\right)\right\|\right\}
$$

where $K$ is compact in the complex numbers $\mathbb{C}$, and $\Pi_{l}$ (resp. $\Pi_{r}$ ) is the left (resp. right) representation of $\mathfrak{B}$. Now consider the set $K(G$; $\mathfrak{B})$ of all continuous functions of $G$ into $\left(\mathfrak{B}, P_{K}\right)$ with compact support. Then, it is a Tomita algebra with the algebraic operations defined by

$$
\begin{aligned}
& (\xi \eta)(g)=\int\left[U_{h^{-1}} \xi\left(g h^{-1}\right)\right] \eta(h) d h \\
& \xi^{\sharp}(g)=U_{g^{-1}} \xi\left(g^{-1}\right)^{\ddagger}, \quad \xi^{b}(g)=U_{g^{-1}} \xi\left(g^{-1}\right)^{b} \\
& \left(\Delta^{z} \xi\right)(g)=\Delta^{z} \xi(g), \quad(J \xi)(g)=J U_{g^{-1}} \xi\left(g^{-1}\right)
\end{aligned}
$$

for $\xi, \eta \in K(G ; \mathfrak{B}), g \in G$ and $z \in \mathbb{C}$. In addition, the left von Neumann algebra $\mathscr{R}_{l}(K(G ; \mathfrak{B}))$ associated with $K(G ; \mathfrak{B})$ is nothing but the crossed product $G \otimes_{\alpha} M$ associated with a triple $(M, G, \alpha)$. Remember that this von Neumann algebra is generated by two kinds of operators $\Pi_{\alpha}(x), \lambda(g)$ $(x \in M, g \in G)$ as follows:

$$
\begin{aligned}
& \left(\Pi_{\alpha}(x) \xi\right)(h)=\alpha_{h^{-1}}(x) \xi(h) \\
& (\lambda(g) \xi)(h)=\xi\left(g^{-1} h\right)
\end{aligned}
$$


for $\xi \in L^{2}(G ; \mathfrak{y})$, where $L^{2}(G ; \mathfrak{y})$ is a Hilbert space of all $\mathfrak{y}$-valued square integrable functions on $G$.

In order to discuss the predual of $G \otimes_{\alpha} M$ in the next section, we need two preliminary lemmas due to J. Phillips [6].

In the present case, the left Hilbert algebra $\mathfrak{N}_{\varphi}$ is obtained from a state, which simplifies the situation. Let $\mathfrak{A}^{\prime}$ be the right Hilbert algebra associated with $\mathfrak{A}_{\varphi}$. Denote by $\mathfrak{B}^{b}$ the closure of the set $\left\{\eta \eta^{b}: \eta \in \mathfrak{Q}^{\prime}\right\}$. Let $L^{1}\left(\mathfrak{2}_{\varphi}\right)$ be the completion of the linear space $V\left(\mathfrak{B}^{b}\right)$ generated by $\Re^{b}$ with respect to the norm $\|\cdot\|_{1}$ defined by

$$
\|\eta\|_{1}=\sup \left\{|<\xi| \eta>\mid: \xi \in \mathfrak{A},\left\|\Pi_{l}(\xi)\right\| \leqq 1\right\}
$$

for $\eta \in V\left(\Re^{b}\right)$.

Then one has the following:

Lemma 2.目。 (i) $L^{1}\left(\mathscr{H}_{\varphi}\right) \cong \mathscr{R}_{l}\left(\mathscr{H}_{\varphi}\right)_{*}$ as a Banach space

(ii) $L^{1}\left(\mathscr{H}_{\varphi}\right)=\left\{\eta \cdot \zeta^{b}: \eta, \zeta \in \mathfrak{y}\right\}$

where $\eta \cdot \zeta^{b}=\|\cdot\|_{1}-\lim \eta_{n} \zeta_{n}^{b}$ for $\eta=\lim \eta_{n}, \zeta=\lim \zeta_{n}\left(\eta_{n}, \zeta_{n} \in \mathfrak{H}^{\prime}\right) . \quad(\mathrm{cf}:[6])$

Let us now take $T$ a non-degenerate \#-representation of a left Hilbert algebra $\mathfrak{A}$ on a Hilbert space $\mathfrak{P}$. This representation $T$ is called square integrable if there exists a cyclic vector $\eta_{0} \in \mathfrak{R}$ for $T$ such that the linear functional $\xi \mapsto\left\langle T(\xi) \eta_{0} \mid \eta_{0}\right\rangle$ is continuous on 2 with respect to Hilbert space norm. Then one gets a criterion for square integrability as follows:

Lemma 2.2. Let $T$ be a square integrable representation of a left Hilbert algebra $\mathfrak{U}$ on a Hilbert space $\mathfrak{R}$. Then there exists a vector $\eta \in \mathfrak{\beta}^{b}$ such that $T$ is unitarily equivalent to a subrepresentation $\Pi_{P}$ of the left representation $\Pi_{l}$ of $\mathfrak{H}$, where $P$ is the projection on the closure of $\Pi_{l}(\mathfrak{O}) \eta$. (cf: [6])

Throughout the paper, we shall adopt the same notations denoted in this section without referring.

\section{§3。 The Predual of Continuous $\mathbb{C}$ rossed Products}

In this section, we shall realize the predual $\left(G \otimes_{\alpha} M\right)_{*}$ of the con- 
tinuous crossed product $G \otimes_{\alpha} M$ constructed from a separable continuous $W^{*}$-dynamical system $(M, G, \alpha)$ as a certain Banach space consisting of functions of $G$ into $M_{*}$. Using this realization, we shall show an extended form of two well-known theorems in harmonic analysis, one of which is a theorem of Gelfand-Raikov, the other is a theorem of Godement.

Given two elements $\eta, \zeta$ in the Tomita algebra $K(G ; \mathfrak{B})$ and $g \in G$, let us define an element $\widetilde{\eta \zeta^{b}}(g)$ in $M_{*}$ by

$$
\widetilde{\eta \zeta^{b}}(g)[x]=\left\langle\eta \zeta^{b}\left(g^{-1}\right)\right| x^{*} \xi_{0}>
$$

for all $x \in M$. Then one computes $\widetilde{\eta \zeta^{b}}(g)\left[\Pi_{l}(\xi)\right]$ as follows:

$$
\begin{aligned}
<\eta \zeta^{b}\left(g^{-1}\right) \mid \xi^{\#}> & =\int_{G}<U_{h}\left[\eta\left(g^{-1} h\right) \zeta(h)^{b}\right] \mid \xi^{\#}>d h \\
& =\int_{G}<U_{h} \eta\left(g^{-1} h\right) \mid \Pi_{\gamma}\left[U_{h} \zeta(h)\right] \xi^{\#}>d h \\
& =\int_{G}<\Pi_{l}(\xi) U_{h} \eta\left(g^{-1} h\right) \mid U_{h} \xi(h)>d h \\
& =\int_{G}<\alpha_{h-1}\left[\Pi_{l}(\xi)\right] \eta\left(g^{-1} h\right) \mid \zeta(h)>d h .
\end{aligned}
$$

Applying (2.3) to (3.2), it follows that

$$
\widetilde{\eta \zeta^{b}}(g)\left[\Pi_{l}(\xi)\right]=<\Pi_{\alpha}\left[\Pi_{l}(\xi)\right] \lambda(g) \eta \mid \zeta>
$$

for all $\zeta \in \mathfrak{B}$. Since $\Pi_{\alpha}$ is ultraweakly continuous on $M$, it implies by (3.3) that

$$
\widetilde{\eta \zeta^{b}}(g)[x]=<\Pi_{\alpha}(x) \lambda(g) \eta \mid \zeta>
$$

for every $x \in M, g \in G$. By definition, it is easily seen that the function $\widetilde{\eta \zeta^{b}}$ is in the space $K\left(G ; M_{*}\right)$ of all norm continuous functions of $G$ into $M_{*}$ with compact support. Define a norm $\|\cdot\|_{\infty}$ on $K\left(G ; M_{*}\right)$ by

$$
\|\Phi\|_{\infty}=\sup \{\|\Phi(g)\|: g \in G\}
$$

for every $\Phi \in K\left(G ; M_{*}\right)$. Then the completion $C_{0}\left(G ; M_{*}\right)$ of $K\left(G ; M_{*}\right)$ with respect to $\|\cdot\|_{\infty}$-norm is nothing but the set of all norm continuous 
$M_{*}$-valued functions on $G$ vanishing at infinity. Since $\widetilde{\eta \zeta^{b}} \in K\left(G ; M_{*}\right)$ for $\eta, \zeta \in K(G ; \mathfrak{B}), \| \widetilde{\eta^{b} \|_{\infty}}$ exists and is estimated as follows:

$$
\left\|\widetilde{\eta \zeta^{b}}\right\|_{\infty} \leqq\left\|\eta \zeta^{b}\right\|_{1}
$$

for all $\eta, \zeta \in K(G ; \mathfrak{B})$. In fact, since $G \otimes_{\alpha} M$ is generated by the set $\left\{\Pi_{\alpha}(x), \lambda(g): x \in M, g \in G\right\}$, it follows from (3.4) that

$$
\begin{aligned}
\widetilde{\left|\eta \zeta^{b}(g)[x]\right|} & =\left|<\Pi_{\alpha}(x) \lambda(g) \eta\right| \zeta>\mid \\
& =\left|\omega_{\eta, \zeta}\left[\Pi_{\alpha}(x) \lambda(g)\right]\right| \\
& \leqq\left\|\omega_{\eta, \zeta}\right\|\|x\|
\end{aligned}
$$

for all $x \in M$ and $g \in G$, where $\left\|\omega_{\eta, \xi}\right\|$ is the uniform norm of the vector state $\omega_{\eta, \zeta}$ on $G \otimes_{\alpha} M$. On the other hand, one has by the definition of $\|\cdot\|_{1}$-norm that

$$
\begin{aligned}
\left\|\eta \zeta^{b}\right\|_{1} & =\sup \left\{|<\xi| \eta \zeta^{b}>\mid: \xi \in K(G ; \mathfrak{B}),\left\|\Pi_{l}(\xi)\right\| \leqq 1\right\} \\
& =\sup \left\{\left|<\Pi_{l}(\xi) \zeta\right| \eta>\mid: \xi \in K(G ; \mathfrak{B}),\left\|\Pi_{l}(\xi)\right\| \leqq 1\right\} \\
& =\left\|\omega_{\eta, \zeta}\right\| .
\end{aligned}
$$

Combining (3.7) and (3.8) together, the inequality (3.6) follows. As a generalization of the scalar case, one can define another norm $\|\cdot\|_{*}$ on the linear space $F_{0}\left(G ; M_{*}\right)$ generated by $\widetilde{\eta \eta^{b}}, \eta \in K(G ; \mathfrak{B})$ as follows:

$$
\|\Phi\|_{*}=\sup \left\{\left|\int_{G} \Phi(g) \circ \Pi_{l}\left[U_{g} \xi(g)\right] d g\right|: \xi \in K(G ; \mathfrak{B}),\left\|\Pi_{l}(\xi)\right\| \leqq 1\right\}
$$

for every $\Phi \in F_{0}\left(G ; M_{*}\right)$. Let $F_{\alpha}\left(G ; M_{*}\right)$ be the completion of $F_{0}\left(G ; M_{*}\right)$ with respect to $\|\cdot\|_{*}$-norm. It is called the Fourier space associated with a triple $(M, G, \alpha)$. In what follows, we shall examine some properties of $F_{\alpha}\left(G ; M_{*}\right)$ exclusively. First of all, since one knows by (3.1) that

$$
\widetilde{4 \eta \zeta^{b}}=\sum_{k=0}^{3} i^{k}\left[\left(\eta+i^{k} \zeta\right)\left(\eta+i^{k} \zeta\right)^{b}\right]^{\sim}
$$

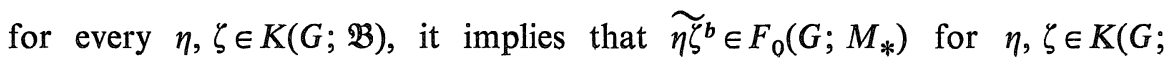
$\mathfrak{B})$. Then, one has that 


$$
\left\|\widetilde{\eta \zeta^{b}}\right\|_{*}=\left\|\eta \zeta^{b}\right\|_{1}
$$

for cvery $\eta, \zeta \in K(G ; \mathfrak{B})$. In fact, applying (3.3) to (3.9),

$$
\left\|\widetilde{\eta \zeta^{b}}\right\|_{*}=\sup \left\{\left|\int_{G}<\Pi_{\alpha} \circ \Pi_{l}\left[U_{g} \xi(g)\right] \lambda(g) \eta\right| \zeta>d g \mid: \xi \in K(G ; \mathfrak{B}),\left\|\Pi_{l}(\xi)\right\| \leqq 1\right\}
$$

On the other hand, it follows from (3.8) that

$$
\left\|\eta \zeta^{b}\right\|_{1}=\sup \left\{|<\xi \eta| \zeta>\mid: \xi \in K(G ; \mathfrak{B}),\left\|\Pi_{l}(\xi)\right\| \leqq 1\right\} .
$$

Using Fubini's theorem and (2.3),

$$
\begin{aligned}
<\xi \eta \mid \zeta> & =\iint_{G \times G}<U_{h^{-1}} \xi\left(g h^{-1}\right) \eta(h) \mid \zeta(g)>d h d g \\
& =\iint_{G \times G}<U_{h^{-1}} \xi(g) \eta(h) \mid \zeta(g h)>d g d h \\
& =\iint_{G \times G}<\left(\Pi_{\alpha} \circ \Pi_{l}[\xi(g)] \eta\right)(h) \mid\left(\lambda(g)^{* \zeta}\right)(h)>d h d g \\
& =\int_{G}<\Pi_{\alpha} \circ \Pi_{l}[\xi(g)] \eta \mid \lambda(g)^{*} \zeta>d g \\
& =\int_{G}<\Pi_{\alpha} \circ \Pi_{l}\left[U_{g} \xi(g)\right] \lambda(g) \eta \mid \zeta>d g,
\end{aligned}
$$

which implies that the equation (3.11) holds. Therefore, it is deduced from (3.6), (3.10) that the Fourier space $F_{\alpha}\left(G ; M_{*}\right)$ is a subspace of $C_{0}\left(G ; M_{*}\right)$. Now given two elements $\eta, \zeta$ in $L^{2}(G ; \mathfrak{y})$, there exist sequences $\left(\eta_{n}\right)_{n},\left(\zeta_{n}\right)_{n}$ of $K(G ; \mathfrak{B})$ which converge to $\eta, \zeta$ in $L^{2}(G ; \mathfrak{y})$ respectively. Hence the sequence $\left\{\eta_{n} \zeta_{n}^{b}\right\}_{n}$ converges to $\eta \cdot \zeta^{b} \in L^{1}(K(G ; \mathfrak{B}))$ with respect to $\|\cdot\|_{1}$-norm. Since the tilde mapping $\sim$ is linear, it follows from (3.11) that the sequence $\left.\widetilde{\eta_{n} \zeta_{n}^{b}}\right\}_{n}$ has a limit point in $F_{\alpha}(G$; $M_{*}$ ) which is denoted by $\widetilde{\eta \cdot \zeta^{b}}$. Then one easily gets that

$$
\widetilde{\eta \cdot \zeta^{b}}(g)[x]=<\Pi_{\alpha}(x) \lambda(g) \eta \mid \zeta>
$$

for all $x \in M, g \in G$. Now remembering Lemma 2.1. (i), the predual $\left(G \otimes_{\alpha} M\right)_{*}$ of the crossed product $G \otimes_{\alpha} M$ associated with a triple $(M, G$, $\alpha)$ is identified with the Banach space $L^{1}(K(G ; \mathfrak{B}))$ of $K(G ; \mathfrak{B})$. Moreover, since $L^{1}(K(G ; \mathfrak{B}))=\left\{\eta \cdot \zeta^{b}: \eta, \zeta \in L^{2}(G ; \mathfrak{y})\right\}$ by Lemma 2.1. (ii), 
it is isometrically isomorphic to the Fourier space $F_{a}\left(G ; M_{*}\right)$ under the tilde $\sim$. Summing up the argument discussed above, we have the following proposition which plays a key role in this paper:

Proposition 3.1. Let $(M, G, \alpha)$ be a separable continuous $W^{*}$ dynamical system. Suppose $M$ is $G$-finite. Then the predual $\left(G \otimes_{\alpha} M\right)_{*}$ of the crossed product $G \otimes_{\alpha} M$ associated with the triple $(M, G, \alpha)$ is isomorphic to the Fourier space $F_{\alpha}\left(G ; M_{*}\right)$ as a Banach space, which is a subspace of $C_{0}\left(G ; M_{*}\right)$. Therefore, $G \otimes_{\alpha} M$ is identified with the dual space $F_{\alpha}\left(G ; M_{*}\right)^{*}$ of $F_{\alpha}\left(G ; M_{*}\right)$.

Let us now consider such elements in $F_{\alpha}\left(G ; M_{*}\right)$ as $\widetilde{\eta \cdot \eta^{b}}, \eta \in \mathbb{L}^{2}(G$; $\mathfrak{y )}$. Then it can be verified that $\widetilde{\eta} \eta^{b}$ has an extended positive definiteness as follows: For an $M_{*}$-valued function $\Phi$ on $G$, it is said to be $\alpha$-positive definite if

$$
\sum_{i, j=1}^{n} \alpha_{g_{j}} \circ \Phi\left(g_{j}^{-1} g_{i}\right)\left[x_{j}^{*} x_{i}\right] \geqq 0
$$

for any finite set $\left(x_{i}\right)_{i=1}^{n}$ in $M$ and $\left(g_{i}\right)_{i=1}^{n}$ in $G$, where $\alpha_{g} \circ \psi(x)=\psi \circ \alpha_{g}^{-1}(x)$ for $\psi \in M_{*}, x \in M$ and $g \in G$. (cf: [12]) In fact, using (3.13),

$$
\begin{aligned}
\sum_{i, j=1}^{n} \alpha_{g_{j}} \circ\left(\eta \cdot \eta^{b}\right)\left(g_{j}^{-1} g_{i}\right)\left[x_{j}^{*} x_{i}\right] & =\sum_{i, j}<\Pi_{\alpha} \circ \alpha_{g_{j}}^{-1}\left(x_{j}^{*} x_{i}\right) \lambda\left(g_{j}^{-1} g_{i}\right) \eta \mid \eta> \\
& =\left\|\sum_{i=1}^{n} \Pi_{\alpha}\left(x_{i}\right) \lambda\left(g_{i}\right) \eta\right\|^{2} \geqq 0 .
\end{aligned}
$$

Thus, any element in $F_{\alpha}\left(G ; M_{*}\right)$ is a linear combination of $\alpha$-positive definite functions. By the similar way as in the scalar case, if $\Phi$ is $\alpha$-positive definite, then one has that

$$
\|\Phi\|_{\infty}=\|\Phi(e)\|, \quad \alpha_{g} \circ \Phi\left(g^{-1}\right)=\Phi(g)^{*}
$$

where $e$ is the unit of $G$, and $\psi^{*}(x)=\overline{\psi\left(x^{*}\right)}$ for $\psi \in M_{*}, x \in M$. Note that in the scalar case, $F_{\alpha}(G ; \mathbb{C})$ is nothing but the Fourier algebra $A(G)$ of $G$ due to $\mathbb{P}$. Eymard. (cf: [3])

We also define an important class of normal representations on von Neumann algebras as follows: Let $(M, G, \alpha)$ be a continuous $W^{*}$ dynamical system. Let $\rho$ be a normal representation of $M$ on a Hilbert space $\mathfrak{R}$. It is called covariant with respect to $\alpha$ if there exists a strongly continuous unitary representation $V$ of $G$ on $\mathfrak{R}$ such that 


$$
V(g) \rho(x) V(g)^{*}=\rho \circ \alpha_{g}(x)
$$

for $x \in M, g \in G$. Then, we specify the relation (3.16) as $(\rho, V) \in \operatorname{Cov}$ rep $(M, G)$. Using two notions cited above, we shall show the following proposition which can be considered as a generalization of GelfandRaikov's theorem.

Proposition 3.2. Lei $(M, G, \alpha)$ be a separable continuous $W^{*_{-}}$ dynamical system. Suppose $M$ is G-finite. Then for any $\alpha$-positive definite function $\Phi$ with norm continuity, there exists a $(\rho, V) \in \operatorname{Cov}$ rep $(M, G)$ on a Hilbert space $\mathfrak{R}$ such that

$$
\Phi(g)[x]=<\rho(x) V(g) \eta_{0} \mid \eta_{0}>_{\Phi}
$$

for every $x \in M$ and $g \in G$, where $\eta_{0} \in$ ? is a cyclic vector for $(\rho, V)$. The converse is also true.

Proof. Denote by $G_{d}$ the group $G$ with the discrete topology. Let $K\left(G_{d} ; \mathfrak{B}\right)$ be the set of all $\mathfrak{B}$-valued functions on $G$ whose support is a finite set. Define a \#-algebra structure on $K\left(G_{d} ; \mathfrak{B}\right)$ by

$$
\begin{aligned}
& (\eta \zeta)(g)=\sum_{h} \eta(h) U_{h} \zeta\left(h^{-1} g\right) \\
& \eta^{\sharp}(g)=U_{g} \eta\left(g^{-1}\right)^{\#} .
\end{aligned}
$$

Since $\Phi$ is $\alpha$-positive definite, one associates a pseudo inner product $<\cdot|\cdot\rangle_{\Phi}$ on $K\left(G_{d} ; \mathfrak{B}\right)$ by the following relation:

$$
<\eta \mid \zeta>_{\Phi}=\sum_{g} \Phi(g) \cup \Pi_{l}\left[\left(\zeta^{\sharp} \eta\right)(g)\right]
$$

Actually, applying (3.18) to (3.19),

$$
\begin{aligned}
<\eta \mid \zeta>_{\Phi} & =\sum_{g, h} \Phi(g) \circ \Pi_{l}\left[U_{h}\left(\zeta\left(h^{-1}\right)^{\sharp} \eta\left(h^{-1} g\right)\right)\right] \\
& =\sum_{g, h} \alpha_{h^{-1}} \circ \Phi(g)\left[\Pi_{l}\left(\zeta\left(h^{-1}\right)\right)^{*} \Pi_{l}\left(\eta\left(h^{-1} g\right)\right)\right] \\
& =\sum_{g, h} \alpha_{h} \circ \Phi\left(h^{-1} g\right)\left[\Pi_{l}(\zeta(h))^{*} \Pi_{l}(\eta(g))\right] .
\end{aligned}
$$

Let $N=\left\{\eta \in K\left(G_{d} ; \mathfrak{B}\right):\|\eta\|_{\Phi}=0\right\} \quad$ where $\|\eta\|_{\Phi}=\langle\eta \mid \eta\rangle_{\Phi}^{1 / 2}$, and $\mathfrak{R}_{0}$ the quotient space of $K\left(G_{d} ; \mathfrak{B}\right)$ by $N$. Moreover, let $\mathfrak{R}$ be the completion of $\mathfrak{R}_{0}$ with respect to $\|\cdot\|_{\Phi^{-} \text {-norm. }}$. Then there exists a strongly continuous 
unitary representation $V$ of $G$ on $\mathfrak{R}$ such that

$$
V(g) \bar{\eta}=\overline{U_{g} \eta_{g}} \quad \text { for } \quad \bar{\eta} \in \mathfrak{R}_{0}
$$

where $\bar{\eta}$ is the equivalence class of $\eta$, and $\left(U_{g} \eta_{g}\right)(h)=U_{g} \eta\left(g^{-1} h\right)$. In fact, one computes by $(3.20)$ that

$$
\begin{aligned}
\|V(g) \bar{\eta}\|_{\Phi}^{2} & =\sum_{h, k} \alpha_{k} \circ \Phi\left(k^{-1} h\right)\left[\Pi_{l}\left(U_{g} \eta_{g}(k)\right)^{*} \Pi_{l}\left(U_{g} \eta_{g}(h)\right)\right] \\
& =\sum_{h, k} \alpha_{g}-1 k^{\circ} \Phi\left(k^{-1} h\right)\left[\Pi_{l}\left(\eta\left(g^{-1} k\right)\right) * \Pi_{l}\left(\eta\left(g^{-1} h\right)\right)\right] \\
& =\|\bar{\eta}\|_{\Phi}^{2}
\end{aligned}
$$

for $\bar{\eta} \in \mathfrak{R}_{0}$. One also estimates that

$$
<V(g) \bar{\eta} \mid \bar{\zeta}>=\sum_{h, k} \alpha_{k} \circ \Phi\left(k^{-1} g h\right)\left[\Pi_{l}(\zeta(k))^{*} \Pi_{l}\left(U_{g} \eta(h)\right)\right]
$$

for $\bar{\eta}, \bar{\zeta} \in \Re_{0}$. Since $\Phi$ is norm continuous and $g \mapsto \alpha_{g}$ is ultrastrong*continuous, it follows from (3.22) that $g \mapsto V(g)$ is strongly continuous. The rest is easily done by direct computation. Furthermore, there exists a normal representation $\rho$ of $M$ on $\Re$ such that

$$
\rho\left[\Pi_{l}(\zeta)\right] \bar{\eta}=\overline{\Pi_{l}(\zeta) \eta}
$$

for $\zeta \in \mathfrak{B}$ and $\bar{\eta} \in \mathfrak{R}_{0}$ where $\left(\Pi_{l}(\zeta) \eta\right)(g)=\Pi_{l}(\zeta) \eta(g)$. In fact, using (3.20),

$$
\begin{aligned}
\left\|\rho\left[\Pi_{l}(\zeta)\right] \bar{\eta}\right\|_{\Phi}^{2} & =\sum_{g, h} \alpha_{h} \circ \Phi\left(h^{-1} g\right)\left[\Pi_{l}\left(\Pi_{l}(\zeta) \eta(h)\right)^{*} \Pi_{l}\left(\Pi_{l}(\zeta) \eta(g)\right)\right] \\
& =\psi_{\eta}\left[\Pi_{l}(\zeta)^{*} \Pi_{l}(\zeta)\right]
\end{aligned}
$$

where $\quad \psi_{\eta}(x)=\sum_{g, h_{h} \alpha_{h} \circ} \Phi\left(h^{-1} g\right)\left[\Pi_{l}(\eta(h))^{*} x \Pi_{l}(\eta(h))\right]$ for $x \in M$. Since $\Phi$ is $\alpha$-positive definite, $\psi_{\eta}$ is a positive element in $M_{*}$. Thus, one gets that

$$
\left\|\rho\left[\Pi_{l}(\zeta)\right] \bar{\eta}\right\|_{\Phi}^{2} \leqq\left\|\Pi_{l}(\zeta)\right\|^{2}\|\bar{\eta}\|_{\Phi}^{2}
$$

for all $\zeta \in \mathfrak{B}$ and $\bar{\eta} \in \mathfrak{R}_{0}$. Given an $x \in M$, there exists a sequence $\left(\zeta_{n}\right)_{n}$ of $\mathfrak{B}$ with $\left\|\Pi_{l}\left(\zeta_{n}\right)\right\| \leqq\|x\|$ such that $\Pi_{l}\left(\zeta_{n}\right)$ converges to $x^{*}$-strongly. By (3.24), there exists a bounded operator $\rho(x)$ on $\mathfrak{R}$ with $\|\rho(x)\| \leqq\|x\|$ such that $\rho\left[\Pi_{l}\left(\zeta_{n}\right)\right]$ converges to $\rho(x)^{*}$-strongly. Then, it is clear that $\rho$ is a *-representation of $M$ on $\mathfrak{R}$. Define $\varepsilon_{0} \in K\left(G_{d} ; \mathfrak{B}\right)$ and $\eta_{0} \in \mathfrak{R}_{0}$ by 


$$
\varepsilon_{0}(g)= \begin{cases}\xi_{0} & (g=e), \quad \eta_{0}=\bar{\varepsilon}_{0} . \\ 0 & (g \neq e) .\end{cases}
$$

Then it is verified that

$$
\Phi(g)\left[\Pi_{l}(\zeta)\right]=<\rho\left[\Pi_{l}(\zeta)\right] V(g) \eta_{0} \mid \eta_{0}>_{\Phi}
$$

for all $\zeta \in \mathfrak{B}$ and $g \in G$. In fact,

$$
\begin{aligned}
<V(g) \eta_{0} \mid \rho\left[\Pi_{l}\left(\zeta^{\sharp}\right)\right] \eta_{0}>_{\Phi} & =\sum_{h . k} \alpha_{k} \circ \Phi\left(k^{-1} h\right)\left[\Pi_{l}\left(\zeta^{\sharp} \varepsilon_{0}(k)\right) * \Pi_{l}\left(U_{g} \varepsilon_{0}\left(g^{-1} h\right)\right)\right] \\
& =\sum_{h} \Phi(h)\left[\Pi_{l}(\zeta) \Pi_{l}\left(U_{g} \varepsilon_{0}\left(g^{-1} h\right)\right)\right] \\
& =\Phi(g)\left[\Pi_{l}(\zeta)\right] .
\end{aligned}
$$

Therefore, since $\rho\left[\Pi_{l}\left(\zeta_{n}\right)\right]$ converges to $\rho(x)^{*}$-strongly, it follows from (3.25) that (3.17) holds. Thus, it implies the normality of $\rho$. In order to prove that $(\rho, V)$ is covariant, it suffices to show that for every $\zeta$ $\in \mathfrak{B}$ and $g \in G$,

$$
V(g) \rho\left[\Pi_{l}(\zeta)\right] V(g)^{*}=\rho \circ \alpha_{g}\left[\Pi_{l}(\zeta)\right]
$$

Computing $V(g) \rho\left[\Pi_{l}(\zeta)\right]$ and $\rho \circ \alpha_{g}\left[\Pi_{l}(\zeta)\right] V(g)$ side by side,

$$
\begin{aligned}
& V(g) \rho\left[\Pi_{l}(\zeta)\right] \bar{\eta}=\overline{U_{g}\left(\Pi_{l}(\zeta) \eta\right)_{g}}=\overline{U_{g} \Pi_{l}(\zeta) \eta_{g}} \\
& \rho \circ \alpha_{g}\left[\Pi_{l}(\zeta)\right] V(g) \bar{\eta}=\overline{\alpha_{g}\left[\Pi_{l}(\zeta)\right] U_{g} \eta_{g}}=\overline{U_{g} \Pi_{l}(\zeta) \eta_{g}}
\end{aligned}
$$

for all $\bar{\eta} \in \mathfrak{R}_{0}$. This proves (3.26). Finally, it is easily seen that $\eta_{0}$ is cyclic for $(\rho, V)$ since $\rho\left[\Pi_{l}(\zeta)\right] V(g) \eta_{0}=\overline{\delta_{g} \otimes \zeta}$ where $\delta_{g}$ is the Dirac function at $g$.

Q.E.D.

Given a norm continuous $\alpha$-positive definite function $\Phi$, we now look for a certain condition under which $\Phi$ belongs to $F_{\alpha}\left(G ; M_{*}\right)$. Define $\left(\Phi \circ \Pi_{l}\right)(g)=\Phi(g) \circ \Pi_{l}$ for $g \in G$ where $\left(\psi \circ \Pi_{l}\right)(\xi)=\psi\left[\Pi_{l}(\xi)\right]\left(\psi \in M_{*}\right.$, $\xi \in \mathfrak{B})$. Assume from now on that

$$
\Phi \circ \Pi_{l} \in L^{2}(G ; \mathfrak{y}) .
$$

in the sense that $\Phi(g) \circ \Pi_{l}$ is continuous on $\mathfrak{B}$ with respect to Hilbert space norm for almost every $g \in G$ and hence can be viewed as an ele- 
ment of $\mathfrak{y}$ and the function $g \mapsto\left(\Phi_{\circ} \Pi_{l}\right)(g)$ is square integrable. There are in fact sufficiently many functions $\Phi$ satisfying (3.27). Put $\Phi^{*}(g)$ $=\Phi\left(g^{-1}\right)^{*}$ for $g \in G$. Then, $\Phi^{*} \circ \Pi_{l} \in \mathbb{L}^{2}(G ; \mathfrak{y})$ if and only if $\Phi \circ \Pi_{l} \in \mathbb{L}^{2}(G$; y) since $\left(\Phi^{*} \circ \Pi_{l}\right)(g)=U_{g^{-1}}\left(\Phi \circ \Pi_{l}\right)(g)$. Applying Proposition 3.2, one has that for $\zeta \in K(G ; \mathfrak{B})$,

$$
\begin{aligned}
<\zeta\left|\Phi^{*} \circ \Pi_{l}\right\rangle & =\int_{G}<\zeta(g) \mid U_{g}^{*}\left(\Phi \circ \Pi_{l}\right)(g)>d g \\
& =\int_{G} \Phi(g)\left[\Pi_{l}\left(U_{g} \zeta(g)\right)\right] d g \\
& =\int_{G}<\rho \circ \Pi_{l}\left[U_{g} \zeta(g)\right] V(g) \eta_{0} \mid \eta_{0}>_{\Phi} d g \\
& =\int_{G}<V(g) \rho \circ \Pi_{l}[\zeta(g)] \eta_{0} \mid \eta_{0}>_{\Phi} d g
\end{aligned}
$$

where $(\rho, V) \in \operatorname{Cov} \operatorname{rep}(M, G)$ on a Hilbert space $\Re$ associated with $\Phi$. Define a \#-representation $T$ of $K(G ; \mathfrak{B})$ on $\mathfrak{R}$ by

$$
T(\zeta)=\int_{G} V(g) \rho \circ \Pi_{l}[\zeta(g)] d g
$$

for $\zeta \in K(G ; \mathfrak{B})$. In fact, for $\eta, \zeta \in K(G ; \mathfrak{B})$,

$$
\begin{aligned}
T(\eta \zeta) & =\iint_{G \times G} V(g) \rho \circ \Pi_{l}\left[U_{h^{-}} \eta\left(g h^{-1}\right) \zeta(h)\right] d h d g \\
& =\iint_{G \times G} V(g h) V\left(h^{-1}\right) \rho \circ \Pi_{l}[\eta(g)] V(h) \rho \circ \Pi_{l}[\zeta(h)] d h d g \\
& =\iint_{G \times G} V(g) \rho \circ \Pi_{l}[\eta(g)] V(h) \rho \circ \Pi_{l}[\zeta(h)] d h d g \\
& =T(\eta) T(\zeta)
\end{aligned}
$$

Similarly, for $\eta \in K(G ; \mathfrak{B})$,

$$
\begin{aligned}
T\left(\eta^{\#}\right) & =\int_{G} V(g) \rho \circ \Pi_{l}\left[U_{g}^{*} \eta\left(g^{-1}\right)^{\#}\right] d g \\
& =\int_{G} \rho \circ \Pi_{l}\left[\eta\left(g^{-1}\right)\right]^{*} V(g) d g
\end{aligned}
$$




$$
\begin{aligned}
& =\int_{G} \rho \circ \Pi_{l}[\eta(g)]^{*} V(g)^{*} d g \\
& =T(\eta)^{*} .
\end{aligned}
$$

By (3.28), this \#-representation $T$ of $K(G ; \mathfrak{B})$ on $\mathfrak{R}$ satisfies the following relation:

$$
<\zeta\left|\Phi^{*} \circ \Pi_{l}>=<T(\zeta) \eta_{0}\right| \eta_{0}>_{\Phi}
$$

for every $\zeta \in K(G ; \mathfrak{B})$. As $\left\langle T(\zeta) \eta_{0}\right| \eta>_{\Phi}=0$ for all $\zeta \in K(G ; \mathfrak{B})$ implies $\eta=0$, it follows from (3.30) and the assumption (3.27) that $T$ is square integrable. Hence, one deduces by Lemma 2.2 that $T$ is equivalent to a subrepresentation $\Pi_{P}$ of $\Pi_{l}$. Since $P$ is a cyclic projection, then one can choose a cyclic vector $\zeta_{0} \in L^{2}(G ; \mathfrak{y})$ for $P$ such that

$$
<T(\zeta) \eta_{0}\left|\eta_{0}>_{\Phi}=<\Pi_{l}(\zeta) \zeta_{0}\right| \zeta_{0}>
$$

for all $\zeta \in K(G ; \mathfrak{B})$. Combining (3.30) and (3.31), it follows from (3.12) that

$$
\int_{G} \Phi^{*}(g)\left[\Pi_{l}(\eta(g))\right] d g=\int_{G}<\Pi_{\alpha^{\circ}} \Pi_{l}\left[U_{g} \eta(g)\right] \lambda(g) \zeta_{0} \mid \zeta_{0}>d g
$$

for all $\eta \in K(G ; \mathfrak{B})$. For any $g \in G$, take $f \in K(G)$ with $f(g) \neq 0$. Consider a sequence $\left(f_{n}\right)_{n}$ of $K(G)$ such that $f_{n}$ converges to $\delta_{g}$ vaguely. Let $\eta_{n}(h)=f_{n}(h) f(h) \xi \in K(G ; \mathfrak{B})$ for any $\xi \in \mathfrak{B}$. Substituting $\eta_{n}$ in (3.32), for $n=1,2, \ldots$,

$$
\int_{G} \Phi^{*}(h)\left[\Pi_{l}(\xi)\right] f(h) f_{n}(h) d h=\int_{G}<\Pi_{\alpha} \circ \Pi_{l}\left[U_{h} \xi\right] \lambda(h) \zeta_{0} \mid \zeta_{0}>f(h) f_{n}(h) d h .
$$

Since $\bar{\Phi}^{*}(h)\left[\Pi_{l}(\xi)\right] f(h)$ and $<\Pi_{\alpha^{\circ}} \Pi_{l}\left[U_{h} \xi\right] \lambda(h) \zeta_{0} \mid \zeta_{0}>f(h)$ are in $K(G)$, one concludes that

$$
\begin{aligned}
\Phi^{*}(g)\left[\Pi_{l}(\xi)\right] & =<\Pi_{\alpha} \circ \Pi_{l}\left[U_{g} \xi\right] \lambda(g) \zeta_{0} \mid \zeta_{0}> \\
& =\widetilde{\zeta_{0} \cdot \zeta_{0}^{b}}(g)\left[\Pi_{l}\left(U_{g} \xi\right)\right],
\end{aligned}
$$

which implies that $\Phi^{*}(g)=\alpha_{g}-10 \widetilde{\zeta_{0} \cdot \zeta_{0}^{b}}(g)$. Therefore, one has by (3.15) that $\left.\Phi^{*}(g)=\widetilde{\zeta_{0} \cdot \zeta_{0}^{b}}\left(g^{-1}\right)^{*}=\widetilde{\left(\zeta_{0} \cdot \zeta_{0}^{b}\right.}\right)^{*}(g)$. So, $\Phi=\widetilde{\zeta_{0} \cdot \zeta_{0}^{b}}$. Summing up the 
argument discussed above, we have the following proposition which would be a generalization of Godement's theorem:

Proposition 3.3. Let $(M, G, \alpha)$ be a separable continuous $W^{*_{-}}$ dynamical system so that $M$ is G-finite. Let $\Phi$ be a norm continuous $\alpha$-positive definite function. If $\Phi_{\circ} \Pi_{l} \in \mathbb{L}^{2}(G ; \mathfrak{y})$, then there exists an element $\zeta_{0} \in \mathbb{L}^{2}(G ; \mathfrak{y})$ such that $\Phi=\widetilde{\zeta_{0} \cdot \zeta_{0}^{b}}$. In this case, $\Phi \in F_{\alpha}\left(G ; M^{*}\right)$.

Remark. In Proposition 3.2 and 3.3, norm continuity of $\Phi$ may be replaced by weak continuity.

\section{§4. Generalized Schwartz Spaces and Fourier Spaces}

In this section, we shall especially study a continuous action of the real numbers $\mathbf{R}$, and try to construct a Fréchet space of vector-valued test functions which generalizes the Schwartz space in the scalar case. Moreover, we shall compare Fréchet seminorms of this space with the norms of the Fourier space introduced in the previous section.

Let $(M, \mathbb{R}, \alpha)$ be a separable continuous $W^{*}$-dynamical system such that $M$ is $\mathbb{R}$-finite. So the results obtained in the previous section are guaranteed. Now define a Fréchet space $S(\mathbb{R} ; \mathfrak{y})$ by the set of all infinite differentiable $\mathfrak{y}$-valued functions $\eta$ on $\mathbb{R}$ such that for every $(p, q) \geqq 0$,

$$
\|\eta\|_{p, q}=\sup _{t \in \mathbf{R}}\left(1+|t|^{p}\right)\left\|D^{q} \eta(t)\right\|<+\infty
$$

where $(p, q) \geqq 0$ means a pair of non-negative integers $p$ and $q, D^{q}$ is the differential operator of order $q$. As in the scalar case, $S(\mathbb{R} ; \mathfrak{y})$ is a dense subspace of $L^{2}(\mathbb{R} ; \mathfrak{y})$.

Let $\left(h_{n}\right)_{n \geqq 0}$ be the sequence of Hermite functions. Namely,

$$
h_{n}(t)=\left(2^{n} n ! \sqrt{\pi}\right)^{-1 / 2} H_{n}(t) e^{-t^{2} / 2} \quad \text { for } \quad n=0,1,2, \ldots
$$

where $H_{n}(t)=(-1)^{n} e^{t^{2}} \frac{d^{n}}{d t^{n}} e^{-t^{2}}$. Let $\left(\xi_{n}\right)_{n \geqq 0}$ be a complete orthonormal system for $\mathfrak{y}$. Then the system $\left(h_{n} \otimes \xi_{m}\right)_{(n, m) \geqq 0}$ is complete orthonormal for $L^{2}(\mathbb{R} ; \mathfrak{y})$. Given an $\eta \in S(\mathbb{R} ; \mathfrak{y})$, then there exists a square summable sequence $\left(C_{n, m}\right)_{(n, m) \geqq 0}$ of complex numbers such that 


$$
\eta=\sum_{(n, m) \geqq 0} C_{n, m}\left(h_{n} \otimes \xi_{m}\right) \quad \text { in } \quad L^{2}(\mathbb{R} ; \mathfrak{y}) .
$$

Using the same ideas as in the scalar case, it can be verified that

$$
N_{p}\left[\left(C_{n, m}\right)\right]^{2}=\sum_{(n, m) \geqq 0}\left|C_{n, m}\right|^{2}(n+1)^{p}<+\infty
$$

for $p=0,1,2, \ldots$ (cf: [7], [5]) Conversely if $\left(C_{n, m}\right)_{(n, m) \geqq 0}$ satisfies (4.4), then the vector $\eta$ defined as in (4.3) belongs to $S(\mathbb{R} ; \mathfrak{y})$. In addition, such correspondence determines an isomorphism between $S(\mathbb{R} ; \mathfrak{y})$ with norms $\|\cdot\|_{p, q}$ and the set of all double sequences $\left(C_{n, m}\right)_{(n, m) \geqq 0}$ satisfying (4.4) with norms $N_{p}(\cdot)$. Let $\Phi$ be a linear combination of norm continuous $\alpha$-positive definite functions $\Phi_{i}$ such that $\Phi_{i} \circ \Pi_{l} \in S(\mathbb{R} ; \mathfrak{y})$. Since $S(\mathbb{R} ; \mathfrak{y})$ is contained in $L^{2}(\mathbf{R} ; \mathfrak{y})$, it follows from Proposition 3.3 that $\Phi \in F_{\alpha}\left(\mathbf{R} ; M_{*}\right)$. Therefore, $\|\Phi\|_{*}$ and $\left\|\Phi \circ \Pi_{l}\right\|_{p, q}$ exist for such $\Phi$ as above. In order to compare them, we need the following lemma:

Lemma 4.1. Let $\left(h_{n}\right)_{n \geqq 0}$ be the sequence of Hermite functions. Then there exists a positive constant $C$ such that

$$
\left\|h_{n}\right\|_{*} \leqq C(n+1) \quad \text { for } \quad n=0,1,2, \ldots,
$$

where $\left\|h_{n}\right\|_{*}$ is the norm of $h_{n}$ in the Fourier algebra $A(\mathbf{R})$ of $\mathbf{R}$.

Proof. Since one knows that

$$
h_{n}(t)=\left(\sqrt{2 \pi} i^{n}\right)^{-1} \int_{\mathbb{R}} e^{i t s} h_{n}(s) d s \quad \text { for } \quad n=0,1, \ldots
$$

it implies by definition that $\left\|h_{n}\right\|_{*}=(\sqrt{2 \pi})^{-1}\left\|h_{n}\right\|_{1}(n=0,1, \ldots)$. Put $g(t)$ $=1+i$. Then one estimates that

$$
\begin{aligned}
\left\|h_{n}\right\|_{1} & \leqq \int_{\mathbf{R}}\left|h_{n}(t) \frac{1}{g(t)}\right| d t+\int_{\mathbf{R}}\left|t h_{n}(t) \frac{1}{g(t)}\right| d t \\
& \leqq\left\|h_{n}\right\|_{2}\left\|\frac{1}{g}\right\|_{2}+\left\|t h_{n}\right\|_{2}\left\|\frac{1}{g}\right\|_{2} .
\end{aligned}
$$

Since $\left\|h_{n}\right\|_{2}=1$ and $\left\|\frac{1}{g}\right\|_{2}=\sqrt{\pi}$, one gets that

$$
\left\|h_{n}\right\|_{1} \leqq \sqrt{\pi}\left(1+\left\|t h_{n}\right\|_{2}\right) \quad \text { for } \quad n=0,1,2, \ldots
$$

As one also knows that 


$$
t h_{n}(t)=\sqrt{\frac{n+1}{2}} h_{n+1}(t)+\sqrt{\frac{n}{2}} h_{n-1}(t) \quad \text { for } n=1,2, \ldots
$$

it follows that $\left\|t h_{n}\right\|_{2}=\sqrt{\frac{2 n+1}{2}}(n=1,2, \ldots)$. Therefore, one obtains that

$$
\begin{aligned}
\left\|h_{n}\right\|_{*} & =(\sqrt{2 \pi})^{-1}\left\|h_{n}\right\|_{1} \\
& \leqq(\sqrt{2})^{-1}\left(1+\sqrt{\frac{2 n+1}{2}}\right) \quad \text { for } n=1,2, \ldots
\end{aligned}
$$

Since $\left\|h_{0}\right\|_{*}=1$, there exists a positive constant $C$ such that

$$
\left\|h_{n}\right\|_{*} \leqq C(n+1) \quad \text { for } n=0,1,2, \ldots \quad \text { Q.E.D. }
$$

Using this lemma, we have the following estimation which is a generalization in the scalar case:

Proposition 4.2. Let $(M, \mathbb{R}, \alpha)$ be a separable continuous $W^{*}$ dynamical system such that $M$ is $\mathbb{R}$-finite. Let $\Phi$ be a linear combination of norm continuous $\alpha$-positive definite functions $\Phi_{i}$ such that $\Phi_{i}$ 。 $\Pi_{l} \in S(\mathbb{R} ; \mathfrak{y})$. Then there exist a positive constant $C$ and $a$ finite set $\left\{\left(p_{i}, q_{i}\right)\right\}_{i=1}^{n}$ which are independent of $\Phi$ such that

$$
\|\Phi\|_{*} \leqq C \max _{1 \leqq i \leqq n}\left\|\Phi \circ \Pi_{l}\right\|_{p_{i}, q_{i}} .
$$

Proof. Since $\Phi \circ \Pi_{l} \in L^{2}(\mathbb{R} ; \mathfrak{y})$, it follows from (4.3) that

$$
\Phi \circ \Pi_{l}=\sum_{n, m} C_{n, m} h_{n} \otimes \xi_{m} \quad \text { in } \quad L^{2}(\mathbb{R} ; \mathfrak{y})
$$

Then one estimates that for any $\xi \in K(\mathbb{R} ; \mathfrak{B})$ with $\left\|\Pi_{l}(\xi)\right\| \leqq 1$,

$$
\begin{aligned}
\left|\int_{\mathbf{R}} \Phi(t) \circ \Pi_{l}\left[U_{t} \xi(t)\right] d t\right| & =\left|\int_{\mathbb{R}}<U_{t} \xi(t)\right| \sum_{n, m} C_{n, m} h_{n}(t) \xi_{m}>d t \mid \\
& \leqq \sum_{n}\left|\int_{\mathbb{R}} h_{n}(t)<U_{t} \xi(t)\right| \sum_{m} C_{n, m} \xi_{m}>d t \mid \\
& =\sum_{n}\left|\int_{\mathbb{R}} h_{n}(t) f_{n}^{\xi}(t) d t\right|
\end{aligned}
$$

where $f_{n}^{\xi}(t)=<U_{t} \xi(t) \mid \sum_{m} C_{n, m} \xi_{m}>\in K(\mathbb{R})$. Since $\left\|\Pi_{l}(\xi)\right\| \leqq 1$,

$$
\left\|\lambda\left(f_{n}^{\xi}\right)\right\| \leqq\left(\sum_{m}\left|C_{n, m}\right|^{2}\right)^{1 / 2} \quad \text { for } \quad n=0,1,2, \ldots
$$


where $\lambda\left(f_{n}^{\xi}\right)=\int_{\mathbf{R}} f_{n}^{\xi}(t) \lambda(t) d t$ and $(\lambda(t) f)(s)=f(s-t)$ for $f \in L^{2}(\mathbb{R})$. Actually, for any $g \in K(\mathbf{R})$ and $t \in \mathbb{R}$,

$$
\begin{aligned}
\left(\lambda\left(f_{n}^{\xi}\right) g\right)(t) & =\int_{\mathbf{R}} f_{n}^{\xi}(s) g(t-s) d s \\
& =\int_{\mathbb{R}}<U_{t-s} \xi(t-s) \mid \sum_{m} C_{n, m} \xi_{m}>g(s) d s \\
& =<\int_{\mathbf{R}} U_{t-s} \xi(t-s) g(s) d s \mid \sum_{m} C_{n, m} \xi_{m}> \\
& =<\left[\Pi_{l}(\xi)\left(g \otimes \xi_{0}\right)\right](t) \mid U_{t}^{*}\left(\sum_{m} C_{n, m} \xi_{m}\right)>
\end{aligned}
$$

Thus, it follows from $\left\|\Pi_{l}(\xi)\right\| \leqq 1$ that

$$
\begin{aligned}
\left\|\lambda\left(f_{n}^{\xi}\right) g\right\|_{2}^{2} & \leqq\left\|\Pi_{l}(\xi)\left(g \otimes \xi_{0}\right)\right\|^{2}\left\|\sum_{m} C_{n, m} \xi_{m}\right\|^{2} \\
& \leqq\left(\sum_{m}\left|C_{n, m}\right|^{2}\right)\|g\|_{2}^{2},
\end{aligned}
$$

which implies (4.8). Using (4.7) and (4.8), one has that

$$
\begin{aligned}
\|\Phi\|_{*} & =\sup \left\{\left|\int_{\mathbf{R}} \Phi(t) \circ \Pi_{l}\left[U_{t} \xi(t)\right] d t\right|: \xi \in K(\mathbb{R} ; \mathfrak{B}),\left\|\Pi_{l}(\xi)\right\| \leqq 1\right\} \\
& \leqq \sup \left\{\sum_{n \geqq 0}\left|\int_{\mathbf{R}} h_{n}(t) f_{n}^{\xi}(t) d t\right|: \xi \in K(\mathbb{R} ; \mathfrak{B}),\left\|\Pi_{l}(\xi)\right\| \leqq 1\right\} \\
& \leqq \sum_{n \geqq 0} \sup \left\{\left|\int_{\mathbb{R}} h_{n}(t) f_{n}^{\xi}(t) d t\right|: \xi \in K(\mathbb{R} ; \mathfrak{B}),\left\|\Pi_{l}(\xi)\right\| \leqq 1\right\} \\
& \leqq \sum_{n \geqq 0} C_{n} \sup \left\{\left|\int_{\mathbf{R}} h_{n}(t) f(t) d t\right|: f \in K(\mathbb{R}),\|\lambda(f)\| \leqq 1\right\} \\
& =\sum_{n \geqq 0} C_{n}\left\|h_{n}\right\|_{*}
\end{aligned}
$$

where $C_{n}=\left[\sum_{m \geqq 0}\left|C_{n, m}\right|^{2}\right]^{1 / 2}$. On the other hand, it follows from Lemma 4.1 that there exists a positive constant $C^{\prime}$ such a that $\left\|h_{n}\right\|_{*} \leqq C^{\prime}(n+1)$ for $n=0,1,2, \ldots$. Hence, one obtains by (4.4) and (4.9) that

$$
\begin{aligned}
\|\Phi\|_{*} & \leqq C^{\prime} \sum_{n=0}^{\infty} \| C_{n}(n+1) \\
& \leqq C^{\prime}\left[\sum_{n=0}^{\infty} C_{n}^{2}(n+1)^{4}\right]^{1 / 2}\left[\sum_{n=0}^{\infty} \frac{1}{(n+1)^{2}}\right]^{1 / 2}
\end{aligned}
$$




$$
\begin{aligned}
& =C^{\prime \prime}\left[\sum_{n, m=0}^{\infty}\left|C_{n, m}\right|^{2}(n+1)^{4}\right]^{1 / 2} \\
& =C^{\prime \prime} N_{4}\left[\left(C_{n, m}\right)\right]
\end{aligned}
$$

where $C^{\prime \prime}=C^{\prime}\left[\sum_{n=1}^{\infty} \frac{1}{n^{2}}\right]^{1 / 2}$. Since $\Phi \circ \Pi_{l} \in S(\mathbb{R} ; \mathfrak{y})$, the observation stated before gives us that there exist a positive constant $C$ and finite family $\left\{\left(p_{i}, q_{i}\right)\right\}_{i=1}^{n}$ such that

$$
C^{\prime \prime} N_{4}\left[\left(C_{n, m}\right)\right] \leqq C \max _{1 \leqq i \leqq n}\left\|\Phi \circ \Pi_{l}\right\|_{p_{i}, q_{i}},
$$

which implies the desired inequality.

Q.E.D.

Now define $\|\Phi\|_{p, q}$ by $\left\|\Phi \circ \Pi_{l}\right\|_{p, q^{*}}$ In what follows, we shall construct a test space of $M_{*}$-valued functions on $\mathbb{R}$ with norms $\|\cdot\|_{p, q}$ which is exactly the Schwartz space in the scalar case.

Let us denote by $\mathfrak{B}_{\infty}$ the set of all elements $\xi \in \mathfrak{B}$ such that $\xi_{U}(t)$ $=U_{t} \xi$ is an infinite differentiable $\left(\mathfrak{B}, P_{K}\right)$-valued function on $\mathbb{R}$. Then, $\mathfrak{B}_{\infty}$ is sufficiently large in $\mathfrak{B}$ since for any $\xi \in \mathfrak{B}$,

$$
\xi_{n}=n^{\frac{1}{2}} \pi^{-\frac{1}{2}} \int_{\mathbb{R}} e^{-n t^{2}} U_{t} \xi d t \in \mathfrak{B}_{\infty} \quad \text { for } \quad n=1,2, \ldots
$$

can be chosen as close to $\xi$ as possible with respect to Hilbert space norm. Moreover, it is a $J$ and $\Delta^{z}$-invariant subalgebra of $\mathfrak{B}$. In fact, concerning the $J$-operation, one sees that for any $\xi \in \mathfrak{B}_{\infty}$,

$$
P_{K}\left[(J)_{U}(t)\right]=P_{K}\left[J \xi_{U}(t)\right]=P_{-K}\left[\xi_{U}(t)\right]
$$

where $-\bar{K}=\{-\bar{z}: z \in K\}$. Thus, $D^{n}(J)_{U}(t)=J\left[D^{n} \xi_{U}(t)\right]$ for $n=0,1$, $2, \ldots$ Concerning the $\Delta^{z}$-operation, one knows that for any $\xi \in \mathfrak{B}_{\infty}$,

$$
P_{K}\left[\left(\Delta^{z} \xi\right)_{U}(t)\right]=P_{K}\left[\Delta^{z} \xi_{U}(t)\right]=P_{K+z}\left[\xi_{U}(t)\right]
$$

where $K+z=\{\omega+z: \omega \in K\}$. Thus, $D^{n}\left(\Delta^{z} \xi\right)_{U}(t)=\Delta^{z}\left[D^{n} \xi_{U}(t)\right]$ for $n=0$, $1,2, \ldots$. Finally concerning multiplication, one sees without difficulty that for any $\xi, \eta \in \mathfrak{B}_{\infty}$,

$$
\begin{aligned}
P_{K}\left[(\xi \eta)_{U}(t)\right] & =P_{K}\left[\xi_{U}(t) \eta_{U}(t)\right] \\
& \leqq P_{K}\left[\xi_{U}(t)\right] P_{K}\left[\eta_{U}(t)\right] .
\end{aligned}
$$


From this continuity of product, one obtains recursively the following equation:

$$
D^{n}(\xi \eta)_{U}(t)=\sum_{k=0}^{n} C_{k} D^{k} \xi_{U}(t) D^{n-k} \eta_{U}(t)
$$

where ${ }_{n} C_{k}=n ! / k !(n-k) !$. Notice that $\mathfrak{B}_{\infty}$ is $U_{t}$-invariant. Now let $C_{c}^{\infty}\left(\mathbb{R} ; \mathfrak{B}_{\infty}\right)$ be the set of all infinite differentiable $\left(\mathfrak{B}_{\infty}, P_{K}\right)$-valued functions on $\mathbf{R}$ with compact support. In order to construct an appropriate algebra sitting in $C_{c}^{\infty}\left(\mathbb{R} ; \mathfrak{B}_{\infty}\right)$ which is invariant under $J$ and $\Delta^{z}$-operations, we shall introduce an operation $L$ on $C_{c}^{\infty}\left(\mathbb{R} ; \mathfrak{B}_{\infty}\right)$ by the following equation:

$$
(L \eta)(s)=D \eta(s)_{U}(0)
$$

for all $\eta \in C_{c}^{\infty}\left(\mathbb{R} ; \mathfrak{B}_{\infty}\right)$ and $s \in \mathbb{R}$. Since $U_{t}\left[D \eta(s)_{U}(0)\right]=D \eta(s)_{U}(t)$ for all $t \in \mathbb{R}, D\left[D \eta(s)_{U}(0)\right]_{U}(0)=D^{2} \eta(s)_{U}(0)$. Thus, $\quad\left(L^{2} \eta\right)(s)=D^{2} \eta(s)_{U}(0)$ formally. By repetition, $\left(L^{k} \eta\right)(s)=D^{k} \eta(s)_{U}(0)$ for $k=0,1,2, \ldots$, where $L^{0} \eta=\eta$. In general, it does not seem to hold that for every $\eta \in C_{c}^{\infty}\left(\mathbb{R} ; \mathfrak{B}_{\infty}\right), L^{k} \eta$ $\in C_{c}^{\infty}\left(\mathbb{R} ; \mathfrak{B}_{\infty}\right)(k=0,1,2, \ldots)$. However, there exist sufficiently many elements $\eta \in C_{c}^{\infty}\left(\mathbb{R} ; \mathfrak{B}_{\infty}\right)$ such that

(i) $\quad L^{k} D^{l} \eta \in C_{c}^{\infty}\left(\mathbb{R} ; \mathfrak{B}_{\infty}\right)$

and

(ii) $D^{l} L^{k} \eta=L^{k} D^{l} \eta$

for every $(k, l) \geqq 0$. In fact, let $\eta=f \otimes \xi$ for $f \in C_{c}^{\infty}(\mathbb{R})$ and $\xi \in \mathfrak{B}_{\infty}$ where $C_{c}^{\infty}(\mathbb{R})$ is the set of all infinite differentiable complex valued functions on $\mathbb{R}$ with compact support. Then, one easily checks that $L^{k} D^{l} \eta=D^{l} f$ $\otimes D^{k} \xi_{U}(0)$, which is in $C_{c}^{\infty}\left(\mathbb{R} ; \mathfrak{B}_{\infty}\right)$ and $L^{k} D^{l} \eta=D^{l} L^{k} \eta$. Let us denote by $C_{U}^{\infty}\left(\mathbb{R} ; \mathfrak{B}_{\infty}\right)$ the set of all elements in $C_{c}^{\infty}\left(\mathbb{R} ; \mathfrak{B}_{\infty}\right)$ satisfying the condition (4.12). We shall show that $C_{U}^{\infty}\left(\mathbb{R} ; \mathfrak{B}_{\infty}\right)$ is a $J$ and $\Delta^{z}$-invariant algebra which is dense in $L^{2}(\mathbb{R} ; \mathfrak{y})$. First of all, concerning to $J$-operation, one computes by repetition that

$$
D^{n} J \eta=(-1)^{n} J\left[\sum_{k=0}^{n}{ }_{n} C_{k} D^{k} L^{n-k}\right] \eta
$$

for every $\eta \in C_{U}^{\infty}\left(\mathbb{R} ; \mathfrak{B}_{\infty}\right)$ and $n=0,1,2, \ldots$. Thus, $J \eta \in C_{c}^{\infty}\left(\mathbb{R} ; \mathfrak{B}_{\infty}\right)$ for all $\eta \in C_{U}^{\infty}\left(\mathbb{R} ; \mathfrak{B}_{\infty}\right)$. Since $J$ commutes with $U_{t}$, one deduces that $L^{k} J \eta$ 
$=J L^{k} \eta$ for all $\eta \in C_{U}^{\infty}\left(\mathbb{R} ; \mathfrak{B}_{\infty}\right)$ and $k=0,1,2, \ldots$ Given an $\eta \in C_{U}^{\infty}(\mathbb{R}$; $\left.\mathfrak{B}_{\infty}\right)$, then, one can check by (4.12) that $D^{l} L^{k} \eta \in C_{U}^{\infty}\left(\mathbb{R} ; \mathfrak{B}_{\infty}\right)$ for any $(k$, $l) \geqq 0$. Therefore, it implies by (4.13) that

$$
L^{m} D^{n} J \eta=(-1)^{n} J\left[\sum_{k=0}^{n} C_{k} D^{k} L^{m+n-k}\right] \eta
$$

for all $(n, m) \geqq 0$. Therefore, $L^{m} D^{n} J \eta \in C_{c}^{\infty}\left(\mathbb{R} ; \mathfrak{B}_{\infty}\right)$ for $(n, m) \geqq 0$. Moreover, since $L^{m} J \eta=J L^{m} \eta$ and $L^{m} \eta \in C_{U}^{\infty}\left(\mathbb{R} ; \mathfrak{B}_{\infty}\right)$, it follows from (4.13), (4.14) that

$$
\begin{aligned}
D^{n} L^{m} J \eta & =D^{n} J L^{m} \eta \\
& =(-1)^{n} J\left[\sum_{k=0}^{n}{ }_{n} C_{k} D^{k} L^{n-k}\right]\left(L^{m} \eta\right) \\
& =L^{m} D^{n} J \eta
\end{aligned}
$$

for all $(n, m) \geqq 0$, which means that $J \eta \in C_{U}^{\infty}\left(\mathbb{R} ; \mathfrak{B}_{\infty}\right)$. Next, concerning to $\Delta^{z}$-operation, one computes that

$$
D^{n} \Delta^{z} \eta=\Delta^{z} D^{n} \eta
$$

for every $\eta \in C_{U}^{\infty}\left(\mathbb{R} ; \mathfrak{B}_{\infty}\right)$ and $n=0,1,2, \ldots$ Thus, $\Delta^{z} \eta \in C_{c}^{\infty}\left(\mathbb{R} ; \mathfrak{B}_{\infty}\right)$ for all $\eta \in C_{U}^{\infty}\left(\mathbb{R} ; \mathfrak{B}_{\infty}\right)$. Since $\Delta^{z}$ commutes with $U_{t}$, one has without difficulty that $L^{k} \Delta^{z} \eta=\Delta^{z} L^{k} \eta$ for all $\eta \in C_{U}^{\infty}\left(\mathbb{R} ; \mathfrak{B}_{\infty}\right)$ and $k=0,1,2, \ldots$ Given an $\eta \in C_{U}^{\infty}\left(\mathbb{R} ; \mathfrak{B}_{\infty}\right)$, then $D^{n} \eta \in C_{U}^{\infty}\left(\mathbb{R} ; \mathfrak{B}_{\infty}\right)$ for $n=0,1,2, \ldots$ Hence, it follows from (4.15) that

$$
L^{m} D^{n} \Delta^{z} \eta=\Delta^{z} L^{m} D^{n} \eta
$$

for all $(n, m) \geqq 0$. Since $L^{m} D^{n} \eta \in C_{U}^{\infty}\left(\mathbb{R} ; \mathfrak{B}_{\infty}\right)$, it implies by (4.16) that $L^{m} D^{n} \Delta^{z} \eta \in C_{c}^{\infty}\left(\mathbb{R} ; \mathfrak{B}_{\infty}\right)$ and $L^{m} D^{n} \Delta^{z} \eta=D^{n} L^{m} \Delta^{z} \eta$ for all $(n, m) \geqq 0$, which means that $\Delta^{z} \eta \in C_{U}^{\infty}\left(\mathbb{R} ; \mathfrak{B}_{\infty}\right)$. Finally, concerning to multiplication, one knows by definition that

$$
(\eta \zeta)(t)=\int_{K} U_{-s} \eta(t-s) \zeta(s) d s
$$

for every $\eta, \zeta \in C_{U}^{\infty}\left(\mathbb{R} ; \mathfrak{B}_{\infty}\right)$ and $t \in \mathbb{R}$, where $K=\operatorname{supp} \zeta$. Let $\xi(t, s)$ $=U_{-s} \eta(t-s) \zeta(s)$. Then, one obtains by repetition that

$$
D_{r}^{n} \xi(t, s)_{U}(r)=\sum_{k=0}^{n}{ }_{n} C_{k} U_{-s} D_{r}^{k} \eta(t-s)_{U}(r) D_{r}^{n-k} \zeta(s)_{U}(r)
$$


for $r, s, t \in \mathbb{R}$ and $n=0,1,2, \ldots$ where the suffix $r$ of $D_{r}$ indicates the variable of differentiation. Since $D_{r}^{n} \eta(s)_{U}(r)=U_{r}\left(L^{n} \eta\right)(s)$ for $\eta \in C_{U}^{\infty}(\mathbb{R}$; $\mathfrak{B}_{\infty}$ ), it follows from (4.18) that

$$
D_{r}^{n} \xi(t, s)_{U}(r)=\sum_{k=0}^{n} C_{k} U_{r}\left[U_{-s}\left(L^{k} \eta\right)(t-s)\left(L^{n-k} \zeta\right)(s)\right] .
$$

Since $\eta, \zeta \in C_{U}^{\infty}\left(\mathbb{R} ; \mathfrak{B}_{\infty}\right)$, one concludes by (4.17), (4.19) that

$$
\begin{aligned}
D_{r}^{n}(\eta \zeta)(t)_{U}(r) & =\int_{K} D_{r}^{n} \xi(t, s)_{U}(r) d s \\
& =\sum_{k=0}^{n}{ }_{n} C_{k} U_{r}\left[\int_{K} U_{-s}\left(L^{k} \eta\right)(t-s)\left(L^{n-k} \zeta\right)(s) d s\right] \\
& =\sum_{k=0}^{n}{ }_{n} C_{k} U_{r}\left[\left(L^{k} \eta\right)\left(L^{n-k} \zeta\right)(t)\right]
\end{aligned}
$$

for $n=0,1,2, \ldots$, which implies that $(\eta \zeta)(t) \in \mathfrak{B}_{\infty}$ for every $t \in \mathbb{R}$ and

$$
L^{n}(\eta \zeta)=\sum_{k=0 n}^{n} C_{k}\left(L^{k} \eta\right)\left(L^{n-k} \zeta\right)
$$

for every $\eta, \zeta \in C_{U}^{\infty}\left(\mathbb{R} ; \mathfrak{B}_{\infty}\right)$. It is deduced by definition that for any $\eta, \zeta \in C_{U}^{\infty}\left(\mathbb{R} ; \mathfrak{B}_{\infty}\right)$

$$
D^{n}(\eta \zeta)=\left(D^{n} \eta\right) \zeta \quad \text { for } \quad n=0,1,2, \ldots
$$

which tells us that $\eta \zeta \in C_{c}^{\infty}\left(\mathbb{R} ; \mathfrak{B}_{\infty}\right)$. It also follows from (4.20) and (4.21) that

$$
\begin{aligned}
L^{n} D^{m}(\eta \zeta) & =L^{n}\left[\left(D^{m} \eta\right) \zeta\right] \\
& =\sum_{k=0}^{n}{ }_{n} C_{k}\left(L^{k} D^{m} \eta\right)\left(L^{n-k} \zeta\right),
\end{aligned}
$$

which implies that $L^{n} D^{m}(\eta \zeta) \in C_{c}^{\infty}\left(\mathbb{R} ; \mathfrak{B}_{\infty}\right)$ since $L^{k} D^{m} \eta$ and $L^{n-k} \zeta$ are in $C_{U}^{\infty}\left(\mathbb{R} ; \mathfrak{B}_{\infty}\right)$ for all $(k, m, n) \geqq 0$. Moreover, it follows from (4.20), (4.21), and (4.22) that

$$
\begin{aligned}
D^{m} L^{n}(\eta \zeta) & =\sum_{k=0}^{n} C_{k} D^{m}\left[\left(L^{k} \eta\right)\left(L^{n-k \zeta}\right)\right] \\
& =\sum_{k=0}^{n} C_{k}\left(D^{m} L^{k} \eta\right)\left(L^{n-k} \zeta\right) \\
& =L^{n} D^{m}(\eta \zeta),
\end{aligned}
$$

which means that $\eta \zeta \in C_{U}^{\infty}\left(\mathbb{R} ; \mathfrak{B}_{\infty}\right)$ for every $\eta, \zeta \in C_{U}^{\infty}\left(\mathbb{R} ; \mathfrak{B}_{\infty}\right)$. Summing 
up the above discussion, we have the following:

Proposition 4.3. Let $(M, \mathbb{R}, \alpha)$ be a separable continuous $W^{*}$ dynamical system such that $M$ is $\mathbb{R}$-finite. Then, there exists a dense $J$ - and $\Delta^{z}$-invariant subalgebra $C_{U}^{\infty}\left(\mathbb{R} ; \mathfrak{B}_{\infty}\right)$ of the Tomita algebra $K(\mathbb{R}$; $\mathfrak{B})$, which is contained in $C_{c}^{\infty}\left(\mathbb{R} ; \mathfrak{B}_{\infty}\right)$, the set of all infinite differentiable $\left(\mathfrak{B}_{\infty}, P_{K}\right)$-valued functions on $\mathbb{R}$ with compact support.

Remark. If $\alpha$ is the trivial action, it is clear that $C_{U}^{\infty}\left(\mathbb{R} ; \mathfrak{B}_{\infty}\right)$ $=C_{c}^{\infty}\left(\mathbb{R} ; \mathfrak{B}_{\infty}\right)$.

By Proposition $4.3, C_{U}^{\infty}\left(\mathbf{R} ; \mathfrak{B}_{\infty}\right)$ is \# and $b$-invariant. Let us define $D_{\alpha}\left(\mathbb{R} ; M_{*}\right)$ the linear space generated by $\widetilde{\eta \eta^{b}}, \eta \in C_{U}^{\infty}\left(\mathbb{R} ; \mathfrak{B}_{\infty}\right)$. Then, this space has the following properties:

Proposition 4.4. Let $(M, \mathbb{R}, \alpha)$ be as in Proposition 4.3. Then one has that

(i) $\Phi \circ \Pi_{l} \in S(\mathbb{R} ; \mathfrak{y})$

and

(ii) $D^{n}\left(\widetilde{\eta \zeta^{b}}\right)=(-1)^{n}\left(\widetilde{\left.D^{n} \eta\right)} \zeta^{b}\right.$

$$
\left(D^{n} \Phi\right) \circ \Pi_{l}=D^{n}\left(\Phi \circ \Pi_{l}\right) \quad(n=0,1,2, \ldots)
$$

for all $\Phi \in D_{\alpha}\left(\mathbb{R} ; M_{*}\right)$ and $\eta, \zeta \in C_{U}^{\infty}\left(\mathbb{R} ; \mathfrak{B}_{\infty}\right)$.

Proof. (i): Using, (3.1), and (2.2) in that order,

$$
\begin{aligned}
\widetilde{\left(\eta \zeta^{b} \circ \Pi_{l}\right)(t)(\xi)} & =<\eta \zeta^{b}(-t) \mid \xi^{\#}> \\
& =<\xi \mid \eta \zeta^{b}(-t)^{b}> \\
& =<\xi \mid U_{t} \zeta \eta^{b}(t)>
\end{aligned}
$$

for all $\eta, \zeta \in C_{U}^{\infty}\left(\mathbb{R} ; \mathfrak{B}_{\infty}\right), \xi \in \mathfrak{B}$, and $t \in \mathbb{R}$. Hence, $\left(\widetilde{\eta \zeta^{b} \circ \Pi_{l}}\right)(t)=U_{t} \zeta^{b}(t)$ for all $t \in \mathbb{R}$. Since $\zeta \eta^{b} \in C_{U}^{\infty}\left(\mathbb{R} ; \mathfrak{B}_{\infty}\right)$ for $\eta, \zeta \in C_{U}^{\infty}\left(\mathbb{R} ; \mathfrak{B}_{\infty}\right)$, one gets by repetition that

$$
D^{n}\left(\widetilde{\eta \zeta}{ }^{b} \circ \Pi_{l}\right)(t)=\sum_{k=0}^{n} C_{k} U_{t}\left(D^{k} L^{n-k} \zeta \eta^{b}\right)(t)
$$


for all $t \in \mathbb{R}$ and $n=0,1,2, \ldots$, which implies that $\widetilde{\eta \zeta^{b}} \Pi_{l} \in C_{c}^{\infty}\left(\mathbb{R} ; \mathfrak{B}_{\infty}\right)$. Therefore, $\widetilde{\eta \zeta^{b}} \circ \Pi_{l} \in S(\mathbb{R} ; \mathfrak{1})$ for every $\eta, \zeta \in C_{U}^{\infty}\left(\mathbb{R} ; \mathfrak{B}_{\infty}\right)$, which yields the statement (i).

(ii): Given $\eta, \zeta \in C_{U}^{\infty}\left(\mathbb{R} ; \mathfrak{B}_{\infty}\right)$. Since $\|\psi\| \leqq\left\|\psi \circ \Pi_{l}\right\|$ for such $\psi \in M_{*}$ as $\left.\psi \circ \Pi_{l} \in 1\right)$ exists, it follows by (i) that $D^{n}\left(\widetilde{\eta \zeta^{b}}\right)$ exists for $n=0,1,2, \ldots$ Moreover, one estimates by (3.3) that

$$
\begin{aligned}
D\left(\widetilde{\eta \xi}^{b}\right)(t)\left[\Pi_{l}(\xi)\right] & =\lim _{r \rightarrow 0}<\Pi_{\alpha} \circ \Pi_{l}(\xi)\left\{\frac{\lambda(t+r)-\lambda(t)}{r}\right\} \eta|\xi\rangle \\
& =\lim _{r \rightarrow 0}\left\langle\left\{\frac{\lambda(r)-I}{r}\right\} \eta \mid \lambda(-t) \Pi_{\alpha} \circ \Pi_{l}\left(\xi^{\sharp}\right) \xi\right\rangle
\end{aligned}
$$

for all $\xi \in \mathfrak{B}$ and $t \in \mathbb{R}$. Since $\eta \in C_{U}^{\infty}\left(\mathbb{R} ; \mathfrak{B}_{\infty}\right), D \eta \in C_{U}^{\infty}\left(\mathbb{R} ; \mathfrak{B}_{\infty}\right)$ and

$$
\begin{aligned}
-(D \eta)(s) & =P_{K}-\lim _{r \rightarrow 0} \frac{\eta(s-r)-\eta(s)}{r} \\
& =P_{K}-\lim _{r \rightarrow 0}\left\{\frac{\lambda(r)-I}{r}\right\} \eta(s)
\end{aligned}
$$

for $s \in \mathbf{R}$. Applying (4.26) to (4.25), one has that

$$
\begin{aligned}
D\left(\widetilde{\eta \zeta^{b}}\right)(t)\left[\Pi_{l}(\xi)\right] & =<-(D \eta) \mid \lambda(-t) \Pi_{\alpha} \circ \Pi_{l}\left(\xi^{\#)}\right) \zeta> \\
& =<\Pi_{\alpha} \circ \Pi_{l}(\xi) \lambda(t)(-1)(D \eta) \mid \zeta>
\end{aligned}
$$

which implies by $(3.3)$ that $D\left(\widetilde{\eta \zeta^{b}}\right)=(-1) \widetilde{(D \eta) \zeta^{b}}$. By repetition, one gets that $D^{n}\left(\widetilde{\eta \zeta^{b}}\right)=(-1)^{n}\left(\widetilde{\left.D^{n} \eta\right) \zeta^{b}}\right.$ for $n=0,1,2, \ldots$. Similarly, using the fact $D^{n} \Phi \in D_{\alpha}\left(\mathbf{R} ; M_{*}\right)$ for every $\Phi \in D_{\alpha}\left(\mathbb{R} ; M_{*}\right)$ and $n=0,1,2, \ldots$, it follows by (i) that $\left(D^{n} \Phi\right) \circ \Pi_{l}$ exists and $\left(D^{n} \Phi\right) \circ \Pi_{l}=D^{n}\left(\Phi \circ \Pi_{i}\right)$ for every $\Phi \in D_{\alpha}$ $\left(\mathbf{R} ; M_{*}\right)$ and $n=0,1,2, \ldots$.

Q.E.D.

Remark. As we saw in (i) of the above proposition, if $\eta \in C_{U}^{\infty}(\mathbb{R}$; $\left.\mathfrak{B}_{\infty}\right),(U \eta)(t)=U_{t} \eta(t) \in C_{U}^{\infty}\left(\mathbb{R} ; \mathfrak{B}_{\infty}\right)$ and $L U \eta=U L \eta, D U \eta=U D \eta+U L \eta$. By Proposition 4.4 (i), norms $\|\cdot\|_{p, q}$ is well-defined on $D_{\alpha}\left(\mathbb{R} ; M_{*}\right)$. Let $S_{\alpha}\left(\mathbf{R} ; M_{*}\right)$ be the completion of $D_{\alpha}\left(\mathbf{R} ; M_{*}\right)$ with respect to $\|\cdot\|_{p, q}$-norms. Since $C_{U}^{\infty}\left(\mathbf{R} ; \mathfrak{B}_{\infty}\right)$ is dense in $L^{2}(\mathbb{R} ; \mathfrak{y})$, and $\left\{\widetilde{\eta \eta^{b}}: \eta \in K(\mathbb{R} ; \mathfrak{B})\right\}$ is total in $F_{\alpha}\left(\mathbb{R} ; M_{*}\right)$, it is verified by Proposition 4.2 that $S_{\alpha}\left(\mathbb{R} ; M_{*}\right)$ is a $\|\cdot\|_{*}$-dense Fréchet space in the Fourier space $F_{\alpha}\left(\mathbb{R} ; M_{*}\right)$. We call it 
a generalized Schwartz space associated with a triple $(M, \mathbb{R}, \alpha)$. By Proposition 4.4 (ii), the $n^{\text {th }}$ differential operator $D^{n}$ on $D_{\alpha}\left(\mathbb{R} ; M_{*}\right)$ is continuous with respect to $\|\cdot\|_{p, q}$-norms. Therefore, the same holds on $S_{\alpha}\left(\mathbf{R} ; M_{*}\right)$. Consequently, combining Propositions $4.2-4.4$, we obtain the following result which is a generalization in the scalar case:

Proposition 4.5. Let $(M, \mathbb{R}, \alpha)$ be a separable continuous $W^{*}$ dynamical system such that $M$ is $\mathbb{R}$-finite. Then, the crossed product $\mathbf{R} \otimes_{\alpha} M$ is a subspace of the dual space $S_{\alpha}\left(\mathbf{R} ; M_{*}\right)^{*}$ of a generalized Schwartz space $S_{\alpha}\left(\mathbb{R} ; M_{*}\right)$ corresponding to $(M, \mathbb{R}, \alpha)$.

Proof. Since $\mathbb{R} \otimes_{\alpha} M$ is the dual Banach space of the Fourier space $F_{\alpha}\left(\mathbb{R} ; M_{*}\right)$, it implies by Proposition 4.2 that the restriction $T \mapsto$ $\left.T\right|_{S_{\alpha}(R ; M *)}$ is a linear isomorphism of $\mathbb{R} \otimes_{\alpha} M$ into $S_{\alpha}\left(\mathbb{R} ; M_{*}\right)^{*}$.

Q.E.D.

Remark. Let $\Phi \in S_{\alpha}\left(\mathbb{R} ; M_{*}\right)$. Then, $\Phi \circ \Pi_{l}$ exists and is in $S(\mathbb{R}$; y). In fact, taking a sequence $\left(\Phi_{n}\right)_{n}$ of $D_{\alpha}\left(\mathbb{R} ; M_{*}\right)$ whose limit point is $\Phi$ with respect to $\|\cdot\|_{p, q}$-norms, we see that there exists an $\eta \in S(\mathbb{R}$; $\mathfrak{y )}$ which is a limit point of $\Phi_{n} \circ \Pi_{l}$ in $S(\mathbb{R} ; \mathfrak{y})$. Therefore, $\langle\xi| \eta(t)>$ $=\lim _{n}<\xi \mid\left(\Phi_{n} \circ \Pi_{l}\right)(t)>=\lim \Phi_{n}(t)\left[\Pi_{l}(\xi)\right]=\Phi(t)\left[\Pi_{l}(\xi)\right] \quad$ for $\quad$ all $\xi \in \mathfrak{B}$ and $t \in \mathbb{R}$. Thus, $\Phi(t) \circ \Pi_{l}$ exists and equals to $\eta(t)$, which means that $\Phi \circ$ $\Pi_{l} \in S(\mathbb{R} ; \mathfrak{y})$.

\section{§5. Fourier Expansions in Crossed Products}

In this section, we shall present certain correspondence of a vector valued function to every element in the space $S_{\alpha}\left(\mathbb{R} ; M_{*}\right)^{*}$ of generalized tempered distributions constructed by a triple $(M, \mathbb{R}, \alpha)$. In particular, a Fourier expansion in crossed products can be obtained in the case of R-finite $W^{*}$-dynamical systems.

Let notation $\left(D_{\alpha}\left(\mathbf{R} ; M_{*}\right), S_{\alpha}\left(\mathbb{R} ; M_{*}\right),\|\cdot\|_{p, q}\right.$, etc. $)$ be as in the previous section. Given an $f \in K(\mathbb{R}) \cup K\left(\mathbb{R}_{d}\right)$, then there exists a $\mathbb{T}_{f \otimes 1} \in S_{\alpha}(\mathbb{R}$; $\left.M_{*}\right)^{*}$ such that

$$
T_{f \otimes 1}(\Phi)=\int_{\mathbb{R}} f(t) \Phi(t)[1] d t
$$


for every $\Phi \in S_{\alpha}\left(\mathbf{R} ; M_{*}\right)$, where $\mathbb{R}_{d}$ is the real numbers with discrete topology. Actually, $\left|T_{f \otimes 1}(\Phi)\right|\|\leqq\| f\left\|_{1}\right\| \Phi \|_{\infty}$. As in the scalar case, to each $\Phi \in D_{\alpha}\left(\mathbf{R} ; M_{*}\right)$, there corresponds a generalized convolution $T_{f \otimes 1} * \Phi$ $\in D_{\alpha}\left(\mathbf{R} ; M_{*}\right)$ of $T_{f \otimes 1}$ and $\Phi$ as follows:

$$
\left(T_{f \otimes 1} * \Phi\right)(t)=\int_{\mathbf{R}} f(s) \Phi(s+t) d s
$$

for all $t \in \mathbb{R}$. Indeed, suppose $\Phi=\widetilde{\eta \eta^{b}}$ for $\eta \in C_{U}^{\infty}\left(\mathbb{R} ; \mathfrak{B}_{\infty}\right)$. Then, one has that

$$
\begin{aligned}
\left(T_{f \otimes 1} * \Phi\right)(t)[x] & =\int_{\mathbf{R}} f(s) \Phi(s+t)[x] d s \\
& =\int_{\mathbf{R}} f(s)<\Pi_{\alpha}(x) \lambda(s+t) \eta|\eta\rangle d s \\
& =<\Pi_{\alpha}(x) \lambda(t) \lambda(f) \eta \mid \eta> \\
& =\widetilde{\xi}^{b}(t)[x]
\end{aligned}
$$

for every $x \in M$ and $t \in \mathbb{R}$, where $\xi=\lambda(f) \eta$. Since $\eta \in C_{U}^{\infty}\left(\mathbb{R} ; \mathfrak{B}_{\infty}\right)$, $\xi \in C_{U}^{\infty}\left(\mathbf{R} ; \mathfrak{B}_{\infty}\right)$. In fact, $L^{n} D^{m} \xi=\lambda(f) L^{n} D^{m} \eta$ for all $(n, m) \geqq 0$. Thus it follows by (5.3) that $T_{f \otimes 1} * \Phi \in D_{\alpha}\left(\mathbb{R} ; M_{*}\right)$. Moreover, since one knows that

$$
D^{q}\left[\left(T_{f \otimes 1} * \Phi\right) \circ \Pi_{l}\right]=\lambda(\bar{f}) D^{q}\left(\Phi \circ \Pi_{l}\right) \quad \text { for } \quad q=0,1,2, \ldots
$$

then one estimates that for any $(p, q) \geqq 0$,

$$
\begin{aligned}
\left\|T_{f \otimes 1} * \Phi\right\|_{p, q} & \leqq\|f\|_{\infty} \int_{K} \sup _{t}\left(1+|t-s|^{p}\right)\left\|D^{q} \Phi \circ \Pi_{l}(t)\right\| d s \\
& \leqq 2^{p}\|f\|_{\infty} \int_{K}\left(1+|s|^{p}\right)\|\Phi\|_{p, q} d s \\
& =C_{f}\|\Phi\|_{p, q}
\end{aligned}
$$

for all $\Phi \in D_{\alpha}\left(\mathbb{R} ; M_{*}\right)$, where $K=\operatorname{supp} f$, and $C_{f}=2^{p}\|f\|_{\infty} \int_{K}\left(1+|s|^{p}\right) d s$. Therefore, it follows that for any $\Phi \in S_{\alpha}\left(\mathbb{R} ; M_{*}\right)$, there exists an element $T_{f \otimes 1} * \Phi$ in $S_{\alpha}\left(\mathbb{R} ; M_{*}\right)$ satisfying (5.2) and (5.4), which enable us to define a convolution product $T * T_{f \otimes 1}$ of $T$ and $T_{f \otimes 1}$ for $T \in S_{\alpha}\left(\mathbb{R} ; M_{*}\right)^{*}$. 
Namely, given a $T \in S_{\alpha}\left(\mathbb{R} ; M_{*}\right)^{*}$ and $f \in K(\mathbb{R}) \cup K\left(\mathbb{R}_{d}\right)$, there exists an element $T * T_{f \otimes 1} \in S_{\alpha}\left(\mathbb{R} ; M_{*}\right)^{*}$ such that $\left(T * T_{f \otimes 1}\right)(\Phi)=T\left[T_{f \otimes 1} * \Phi\right]$ for every $\Phi \in S_{\alpha}\left(\mathbb{R} ; M_{*}\right)$. By the similar way as in the scalar case, it implies by (5.4) that there exist a positive constant $C$ and a $(p, q) \geqq 0$ such that

$$
\left|\left(T * T_{f \otimes 1}\right)(\Phi)\right| \leqq C\|\Phi\|_{p, q}
$$

for all $\Phi \in S_{\alpha}\left(\mathbb{R} ; M_{*}\right)$. (cf: [11]) The $(p, q) \geqq 0$ depends only on $T$.

Now let us assume that $f \in C_{c}^{\infty}(\mathbb{R})$. Then, one easily computes that for any $T \in S_{\alpha}\left(\mathbb{R} ; M_{*}\right)^{*}$ and $\Phi \in S_{\alpha}\left(\mathbb{R} ; M_{*}\right)$

$$
\begin{aligned}
\left(T * T_{f \otimes 1}\right)(\Phi) & =T\left[T_{f \otimes 1} * \Phi\right] \\
& =T\left[\int_{\mathbf{R}} f(s)(\lambda(-s) \Phi) d s\right] \\
& =T\left[\int_{\mathbf{R}}(\lambda(s) \check{f}) \otimes \Phi(s) d s\right],
\end{aligned}
$$

where $\quad(\lambda(-s) \Phi)(t)=\Phi(t+s),(\lambda(s) \check{f})(t)=f(s-t) \quad$ and $\quad[(\lambda(s) \check{f}) \otimes \Phi(s)](t)=$ $(\lambda(s) \check{f})(t) \Phi(s)$. Since $f \in C_{c}^{\infty}(\mathbb{R})$, it follows that

(i) $[(\lambda(s) \check{f}) \otimes \Phi(s)] \circ \Pi_{l} \in S(\mathbb{R} ; \mathfrak{y})$

and

(ii) $\left\|[(\lambda(s) \check{f}) \otimes \Phi(s)] \circ \Pi_{l}\right\|_{p, q} \leqq 2^{p}\left(1+|s|^{p}\right)\left\|\Phi(s) \circ \Pi_{l}\right\|\|f\|_{p, q}$

for all $(p, q) \geqq 0$ and $s \in \mathbb{R}$, where $\|f\|_{p, q}=\sup _{t \in \mathbb{R}}\left(1+|t|^{p}\right)\left|D^{q} f(t)\right|$. However, it would be doubtful in general that $(\lambda(s) \check{f}) \otimes \Phi(s) \in S_{\alpha}\left(\mathbb{R} ; M_{*}\right)$ for $\Phi \in S_{\alpha}\left(\mathbb{R} ; M_{*}\right)$. Thus, in order to analyze (5.6), let us introduce a new space $S_{\Pi_{l}}\left(\mathbb{R} ; M_{*}\right)$, the set of all infinite differentiable $M_{*}$-valued functions $\Phi$ of $\mathbb{R}$ such that $\Phi \circ \Pi_{l} \in S(\mathbb{R} ; \mathfrak{y})$ with $\|\cdot\|_{p, q}$-norms. It is a Fréchet space containing $S_{\alpha}\left(\mathbb{R} ; M_{*}\right)$ as a closed subspace. By the HahnBanach extension theorem, there exists an element $T^{\prime} \in S_{\Pi_{l}}\left(\mathbb{R} ; M_{*}\right)$ for every $T \in S_{\alpha}\left(\mathbb{R} ; M_{*}\right)^{*}$ such that

$$
T\left[\int_{\mathbf{R}}(\lambda(s) \breve{f}) \otimes \Phi(s) d s\right]=\int_{\mathbf{R}} T^{\prime}[(\lambda(s) \breve{f}) \otimes \Phi(s)] d s
$$

for every $\Phi \in S_{\alpha}\left(\mathbb{R} ; M_{*}\right)$. Consider now a bounded conjugate linear functional $u_{s}$ on $\mathfrak{l}$ for $s \in \mathbb{R}$ as follows: 


$$
u_{s}(\xi)=T^{\prime}\left[(\lambda(s) \check{f}) \otimes \psi_{\xi}\right]
$$

for every $\xi \in \mathfrak{1}$, where $\psi_{\xi} \in M_{*}$ is as $\langle\zeta \mid \xi\rangle=\psi_{\xi}\left[\Pi_{l}(\zeta)\right]$ for $\zeta \in \mathfrak{B}$. In fact, using the same way as to get (5.7), one gets by (5.9) that there exist a $C^{\prime}>0$ such that

$$
\left|u_{s}(\xi)\right| \leqq C^{\prime}\left(1+|s|^{p}\right)\|f\|_{p, q}\|\xi\|,
$$

which guarantees that there exists a unique element $\eta_{f}(s) \in \mathfrak{y}$ such that

$$
\text { (i) } \quad\left\|\eta_{f}(s)\right\| \leqq C^{\prime}\left(1+|s|^{p}\right)\|f\|_{p, q}
$$

and

(ii) $u_{s}(\xi)=\left\langle\eta_{f}(s)\right| \xi>\quad$ for all $\xi \in \mathfrak{y}$.

Since $f \in C_{c}^{\infty}(\mathbf{R})$, the function $s \mapsto \eta_{f}(s)$ is weakly infinite differentiable 1)-valued function on $\mathbf{R}$. Thus, it is strongly infinite differentiable. (cf: [4]) Combining (5.6)-(5.10) altogether, one concludes that there exist a $(p, q) \geqq 0$, a $C>0$, and a $\eta_{f} \in C^{\infty}(\mathbf{R} ; \mathfrak{y})$ such that

$$
\text { (i) }\left\|\eta_{f}(t)\right\| \leqq C\left(1+|t|^{p}\right)\|f\|_{p, q}
$$

and

(ii) $\left(T * T_{f \otimes 1}\right)(\Phi)=\int_{\mathbf{R}}<\eta_{f}(s) \mid \Phi(s) \circ \Pi_{l}>d s$

for every $\Phi \in S_{\alpha}\left(\mathbb{R} ; M_{*}\right)$ and $t \in \mathbb{R}$.

Note that the above pair $(p, q) \geqq 0$ is independent of the choice of $f$, and the constant $C$ is dependent upon supp $f$. Let us now take $W$ a bounded open set in $\mathbb{R}$ containing zero $0 \in \mathbb{R}$. By the parametrix formula in the scalar case, to the above $q$, there correspond a positive integer $r$, an element $g \in C^{\infty}(W)$, and an element $h \in C^{q}(W)$ such that

$$
f(0)=\int_{\mathbf{R}} h(t) D^{r} f(t) d t+\int_{\mathbf{R}} g(t) f(t) d t
$$

for every $f \in S(\mathbf{R})$, where $C^{\infty}(W)$ [resp. $C^{q}(W)$ ] is the set of all infinite [resp. $q$-times] differentiable complex valued functions whose support is contained in $W$, and $S(\mathbb{R})$ is the Schwartz space of $\mathbb{R}$. (cf: [11]) Thus it follows from (5.2) and (5.12) that 


$$
T_{\delta_{0} \otimes 1} * \Phi=T_{h \otimes 1} * D^{r} \Phi+T_{g \otimes 1} * \Phi
$$

for every $\Phi \in S_{\alpha}\left(\mathbf{R} ; M_{*}\right)$. Since $T * T_{\delta_{0} \otimes 1}=T$, one has by (5.13) that

$$
T=D^{r} T * T_{h \otimes 1}+T * T_{g \otimes 1}
$$

where $\left(D^{r} T\right)(\Phi)=T\left(D^{r} \Phi\right)$ for $\Phi \in S_{a}\left(\mathbb{R} ; M_{*}\right)$. Now choose a sequence $\left(k_{n}\right)_{n}$ of $C^{\infty}(W)$ such that $\left\|k_{n}-h\right\|_{i, j} \rightarrow 0$ as $n \rightarrow \infty$ for $i=0, \ldots, p, j=0, \ldots, q$. By (5.4),

$$
\begin{aligned}
\left\|T_{k_{n} \otimes 1} * D^{r} \Phi-T_{h \otimes 1} * D^{r} \Phi\right\|_{p, q} & \leqq C_{k_{n}-h}\left\|D^{r} \Phi\right\|_{p, q} \\
& =2^{p}\|\Phi\|_{p, q+r}\left(\int_{W}(1+|s|)^{p} d s\right)\left\|k_{n}-h\right\|_{\infty},
\end{aligned}
$$

which implies that $D^{r} T * T_{k_{n} \otimes 1}$ converges to $D^{r} T * T_{h \otimes 1}$ in $S_{\alpha}\left(\mathbb{R} ; M_{*}\right)^{*}$. On the other hand, since $k_{n} \in C^{\infty}(W)$ for $n=0,1,2, \ldots$, there exist a $C^{\prime}>0$ and a $\eta_{k_{n}} \in C^{\infty}(\mathbb{R} ; \mathfrak{y})$ such that

$$
\text { (i) } \quad\left\|\eta_{k_{n}}(t)\right\| \leqq C^{\prime}\left(1+|t|^{p}\right)\left\|k_{n}\right\|_{p, q}
$$

and

$$
\text { (ii) } \quad\left(D^{r} T * T_{k_{n} \otimes 1}\right)(\Phi)=\int_{\mathbb{R}}<\eta_{k_{n}}(s) \mid D^{r} \Phi(s) \circ \Pi_{l}>d s
$$

for every $\Phi \in S_{\alpha}\left(\mathbb{R} ; M_{*}\right)$ and $t \in \mathbb{R}$. One knows by construction that

$$
\left\|\eta_{k_{n}}(t)-\eta_{k_{m}}(t)\right\| \leqq C^{\prime}\left(1+|t|^{p}\right)\left\|k_{n}-k_{m}\right\|_{p, q}
$$

for every $t \in \mathbb{R}$, which tells us by (5.15) that there exists a $\eta_{h} \in C(\mathbb{R}$; y) such that

$$
\text { (i) }\left\|\eta_{h}(t)\right\| \leqq C^{\prime}\left(1+|t|^{p}\right)\|h\|_{p, q}
$$

and

(ii) $\quad\left(D^{r} T * T_{h \otimes 1}\right)(\Phi)=\int_{\mathbb{R}}<\eta_{h}(s) \mid D^{r} \Phi(s) \circ \Pi_{l}>d s$

for every $\Phi \in S_{\alpha}\left(\mathbb{R} ; M_{*}\right)$. Combining (5.11) with $f$ replacing $g$, (5.15) and (5.16), one gets that

$$
T(\Phi)=\int_{\mathbf{R}}\left\{\left\langle\eta_{h}(s) \mid D^{r} \Phi(s) \circ \Pi_{l}\right\rangle+<\eta_{g}(s)\left|\Phi(s) \circ \Pi_{l}\right\rangle\right\} d s
$$


for every $\Phi \in S_{\alpha}\left(\mathbb{R} ; M_{*}\right)$. Let us define $\zeta_{g} \in C^{\infty}(\mathbb{R} ; \mathfrak{y})$ by

$$
\zeta_{g}(t)=\int_{0}^{t} \int_{0}^{t_{r-1}} \cdots \int_{0}^{t_{1}}(-1)^{r} \eta_{g}(s) d s d t_{1} \ldots d t_{r-1}
$$

Since $\left\|\eta_{g}(s)\right\| \leqq C\left(1+|s|^{p}\right)\|g\|_{p, q}$, it follows from (5.18) that there exists a $C^{\prime \prime}>0$ such that $\left\|\zeta_{g}(t)\right\| \leqq C^{\prime \prime}\left(1+|t|^{p+r}\right)$ for all $t \in \mathbb{R}$. Moreover, $D^{r} \zeta_{g}$ $=(-1)^{r} \eta_{g}$. Thus, one concludes that

$$
\begin{aligned}
\int_{\mathbf{R}}<\zeta_{g}(s)\left|D^{r} \Phi(s) \circ \Pi_{l}\right\rangle d s & =\int_{\mathbb{R}}<(-1)^{r} D^{r} \zeta_{g}(s) \mid \Phi(s) \circ \Pi_{l}>d s \\
& =\int_{\mathbb{R}}<\eta_{g}(s) \mid \Phi(s) \circ \Pi_{l}>d s,
\end{aligned}
$$

which implies by (5.17) that

$$
T(\Phi)=\int_{\mathbf{R}}<\eta_{h}(s)+\zeta_{g}(s) \mid D^{r} \Phi(s) \circ \Pi_{l}>d s
$$

for every $\Phi \in S_{\alpha}\left(\mathbf{R} ; M_{*}\right)$. The condition that $\left\|\eta_{h}(t)\right\| \leqq C^{\prime}\|h\|_{p, q}\left(1+|t|^{p}\right)$ and $\left\|\zeta_{g}(t)\right\| \leqq C^{\prime \prime}\left(1+|t|^{p+r}\right)$ gives us that there exists a $\xi \in B C(\mathbb{R} ; \mathfrak{y})$ such that

$$
T(\Phi)=\int_{\mathbf{R}}\left(1+|s|^{p+r}\right)<\xi(s) \mid D^{r} \Phi(s) \circ \Pi_{l}>d s
$$

for every $\Phi \in S_{\alpha}\left(\mathbf{R} ; M_{*}\right)$, where $B C(\mathbf{R} ; \mathfrak{y})$ is the set of all bounded continuous $\mathfrak{y}$-valued functions on $\mathbb{R}$. Conversely, suppose that there exist a $(p, q) \geqq 0$ and a $\xi \in B C(\mathbb{R} ; \mathfrak{y})$ such that

$$
T(\Phi)=\int_{\mathbb{R}}\left(1+|s|^{p}\right)<\xi(s) \mid D^{q} \Phi(s) \circ \Pi_{l}>d s \quad \text { for } \quad \Phi \in S_{\alpha}\left(\mathbb{R} ; M_{*}\right) .
$$

Then, one estimates that

$$
\begin{aligned}
|T(\Phi)| & \leqq \int_{\mathbf{R}}\left(1+|s|^{p}\right)\|\xi(s)\|\left\|D^{q} \Phi(s) \circ \Pi_{l}\right\| d s \\
& \leqq C\|\Phi\|_{r, q} \int_{\mathbf{R}}\left(1+|s|^{p}\right)\left(1+|s|^{r}\right)^{-1} d s
\end{aligned}
$$

for some $C>0$ and $r=1,2, \ldots$ Since $\int_{\mathbb{R}}\left(1+|s|^{p}\right)(1+|s|)^{-1} d s$ is finite 
for a sufficiently large number $r$, we see that $T \in S_{\alpha}\left(\mathbb{R} ; M_{*}\right)^{*}$. Summing up the argument discussed above, we have the following theorem which is a generalization of the Schwartz's theorem for tempered distributions. (cf: [7], [11])

Theorem 5.1. Let $(M, \mathbb{R}, \alpha)$ be a separable continuous $W^{*}$-dynamical system so that $M$ is $\mathbb{R}$-finite. Let $S_{\alpha}\left(\mathbf{R} ; M_{*}\right)^{*}$ be the dual space of a generalized Schwartz space $S_{\alpha}\left(\mathbb{R} ; M_{*}\right)$ with respect to $(M, \mathbb{R}, \alpha)$. Then, $T \in S_{\alpha}\left(\mathbb{R} ; M_{*}\right)^{*}$ if and only if there exist $a(p, q) \geqq 0$ and $a \xi$ $\in B C(\mathbb{R} ; \mathfrak{y})$ such that

$$
T(\Phi)=\int_{\mathbb{R}}\left(1+|s|^{p}\right)<\xi(s) \mid D^{q} \Phi(s) \circ \Pi_{l}>d s
$$

for every $\Phi \in S_{\alpha}\left(\mathbb{R} ; M_{*}\right)$.

As we have shown in Proposition 4.5 , the crossed product $\mathbb{R} \otimes_{\alpha} M$ associated with $(M, \mathbb{R}, \alpha)$ is contained in $S_{\alpha}\left(\mathbb{R} ; M_{*}\right)^{*}$. Let $T \in S_{\alpha}(\mathbb{R}$; $\left.M_{*}\right)^{*}$ as in (5.21). In what follows, we shall look for a certain condition of the triple $(\xi, p, q)$ under which $T \in \mathbb{R} \otimes_{\alpha} M$. Let us denote by $S_{U}(\mathbb{R} ; \mathfrak{y})$ the set of all elements $\Phi \circ \Pi_{l}, \Phi \in S_{\alpha}\left(\mathbb{R} ; M_{*}\right)$. By Proposition 4.4 (ii), $S_{U}(\mathbb{R} ; \mathfrak{y})$ is a $D$-invariant closed subspace of $S(\mathbb{R} ; \mathfrak{y})$. Let $\eta$ be a $\mathfrak{y}$-valued function on $\mathbb{R}$. Then, it is called slowly increasing if there exist a non-negative integer $p$ and a $\xi \in B C(\mathbb{R} ; \mathfrak{y})$ such that $\eta(t)=\left(1+|t|^{p}\right) \xi(t)$. For such a function $\eta$, there exists a $T_{\eta}$ in the dual space $S_{U}(\mathbb{R} ; \mathfrak{y})^{*}$ of $S_{U}(\mathbb{R} ; \mathfrak{y})$ such that

$$
T_{\eta}(\zeta)=\int_{\mathbb{R}}<\eta(s) \mid \zeta(s)>d s \quad \text { for all } \quad \zeta \in S_{U}(\mathbb{R} ; \mathfrak{y})
$$

Then, the equation (5.21) means that

$$
T(\Phi)=D^{q} T_{\eta}\left(\Phi \circ \Pi_{l}\right)
$$

for every $\Phi \in S_{\alpha}\left(\mathbb{R} ; M_{* *}\right)$, where $\left(D^{q} T_{\eta}\right)(\zeta)=T_{\eta}\left(D^{q} \zeta\right)$ for $\zeta \in S_{U}(\mathbb{R} ; \mathfrak{y})$. Now assume that $T \in \mathbb{R} \otimes_{\alpha} M$. Then, there exists a positive constant $C$ such that $|T(\Phi)| \leqq C\|\Phi\|_{*}$ for every $\Phi \in S_{\alpha}\left(\mathbb{R} ; M_{*}\right)$. Remembering the equation (3.9) together with Remark after Proposition 4.3, one obtains that 


$$
\|\Phi\|_{*}=\sup \left\{\left|T_{U \zeta}\left(\Phi \circ \Pi_{l}\right)\right|: \zeta \in K(\mathbb{R} ; \mathfrak{B}),\left\|\Pi_{l}(\zeta)\right\| \leqq 1\right\}
$$

Since $|T(\Phi)| \leqq C\|\Phi\|_{*}$, it follows from (5.22) and (5.23) that

$$
\left|D^{q} T_{\eta}(\omega)\right| \leqq \sup \left\{\left|T_{U \zeta}(\omega)\right|: \zeta \in K(\mathbb{R} ; \mathfrak{B}),\left\|\Pi_{l}(\zeta)\right\| \leqq C\right\}
$$

for every $\omega \in S_{U}(\mathbb{R} ; \mathfrak{y})$. Since $E=\left\{T_{U \zeta}: \zeta \in K(\mathbb{R} ; \mathfrak{B}),\left\|\Pi_{l}(\zeta)\right\| \leqq C\right\}$ is a circled convex subset of $\left.S_{U}(\mathbf{R} ; \mathfrak{y})\right)^{*}$, one deduces by $(5.24)$ that $D^{q} T_{\eta}$ is in the weak* closure $F$ of $E$. Hence $D^{q} T_{\eta}$ is a limit point of $T_{U \zeta_{l}}$, $\left\|\Pi_{l}\left(\zeta_{l}\right)\right\| \leqq C$ with respect to weak* topology. The converse is also valid. Consequently, we have the following main theorem:

Theorem 5.2. Let $(M, \mathbb{R}, \alpha)$ be as in Theorem 5.1, and $T \in S_{\alpha}(\mathbb{R}$; $\left.M_{*}\right)^{*}$. Then

(1) There exists $\hat{T} \in S_{U}(\mathbb{R} ; \mathfrak{y})^{*}$ such that

$$
T(\Phi)=\widehat{T}\left(\Phi \circ \Pi_{l}\right)
$$

for every $\Phi \in S_{\alpha}\left(\mathbf{R} ; M_{*}\right)$,

(2) $\hat{T}=D^{p} T_{\eta}$ for some integer $p$ and slowly increasing continuous $\mathfrak{y}$ valued function $\eta$.

(3) $T \in \mathbb{R} \otimes_{\alpha} M$ if and only if there exists a net of $\zeta_{l} \in K(\mathbb{R} ; \mathfrak{B})$ such that

$$
\hat{T}=\lim T_{U \zeta_{l}}
$$

where $\sup \left\|\Pi_{l}\left(\zeta_{l}\right)\right\|<\infty$.

Remark. One may find a prototype of the above theorem in G. Loupias and S. Miracle-Sole's paper [5], which tells us that any operator of the Schrödinger representation can be considered as a tempered distribution on the phase space of a system with $n$ degrees of freedom.

Remark. Let us remember the definition of $\Pi_{\alpha}(x), \lambda(t)(x \in M, t \in \mathbb{R})$ which are generators of $\mathbb{R} \otimes_{\alpha} M$. Then, one easily computes that $\Pi_{\alpha}$ 。 $\Pi_{l}(\eta)[\Phi]=T_{\delta_{0} \otimes \eta}\left(\Phi \circ \Pi_{l}\right), \lambda(t)[\Phi]=T_{\delta_{t} \otimes \xi_{0}}\left(\Phi \circ \Pi_{l}\right)$ for every $\eta \in \mathfrak{B}$ and $\Phi \in S_{\alpha}(\mathbb{R}$; $\left.M_{*}\right)$.

Corollary 5.3. Let $T \in S_{\alpha}\left(\mathbb{R} ; M_{*}\right)^{*}$ be as in Theorem 5.2. Then, it is $\|\cdot\|_{*}$-continuous if and only if there exists a unique element $T_{0} \in \mathbb{R}$ $\otimes_{\alpha} M$ such that 


$$
\left.<T_{0} \eta_{1} \mid \eta_{2}>=D^{p} T_{\eta}\left(\widetilde{\eta_{1} \eta_{2}^{b}} \circ \Pi_{l}\right)=T \widetilde{\left(\eta_{1} \eta_{2}^{b}\right.}\right)
$$

for every $\eta_{i} \in C_{U}^{\infty}\left(\mathbf{R} ; \mathfrak{B}_{\infty}\right), i=1,2$.

\section{References}

[1] Connes, A., Une classification des facteurs de type III. Ann. Sci. École Norm. Sup., 4e série, 6 fasc 2 (1973).

[2] Connes, A., Almost periodic states and factors of type $\mathrm{III}_{1} . J$. Functional Analysis, 16 (1974), 415-445.

[3] Eymard, P., L'algèbre de Fourier d'un groupe localement compact. Bull. Soc. Math. France, 92 (1964), 181-236.

[4] Grothendieck, A., Produits tensoriels topologiques et espaces nucléaires. Mem. Amer. Math. Soc., 16 (1955).

[5] Loupias, G. et Miracle-Sole, S., C*-algèbres des systems canoniques. II Ann. Inst. Henri Poincaré, 6 (1967), 39-58.

[6] Phillips, J., Positive integrable elements relative to a left Hilbert algebra. J. Functional Analysis, 13 (1973), 390-409.

[7] Schwartz, L., Théorie des distributions. Gauthier-Villars, Paris (1966).

[8] Takai, H., The quasi-orbit space of continuous $\mathrm{C}^{*}$-dynamical systems. Preprint (1974), CPT-CNRS, Marseille.

[9] Takesaki, M., Duality for crossed products and the structure of von Neumann algebras of type III. Acta. Math., 131 (1973) 249-310.

[10] Tomita, M., Standard forms of von Neumann algebras. The 5-th Func. Anal. Symp. of the Math. Soc. of Japan, Sendai (1967).

[11] Yosida, K., The theory of distributions (in Japanese). Kyôritsu, Tokyo, Japan (1968).

[12] Zeller-Meier, G., Produits croisés d'une $\mathrm{C}^{*}$-algèbre par un groupe d'automorphismes. J. Math. Pures Appl., 47 (1968), 101-239.

[13] Araki, H., Structure of some von Neumann algebras with isolated discrete modular spectrum, Publ. RIMS, Kyoto Univ., 9 (1973), 1-44.

[14] Takesaki, M., The structure of von Neumann algebra with a homogeneous periodic state. Acta Math., 131 (1973), 79-121. 